\title{
Elements, structure and dynamics of the mind-brain system A model based on 'Cognitive Feature Detectors'
}

\begin{abstract}
General principles of a unified theory of cognition and its relationships with the brain are outlined, integrating insights from several existing theories and approaches. Structure, dynamics, and development of the neural/cognitive system are described. Cognitive Feature Detectors (CFDs) are the fundamental units: they have a (minimalist) semantic value, strictly related to their location in a cognitive space. A cognitivo-neural isomorphism is postulated between CFDs in this cognitive space, and single (or small sets of) neurons in the neural network. The cognitive space is made of two parts (executive and perceptive), densely connected, both hierarchically organized and clustered in cognitive maps. Within maps, inhibitory connections implement competition between incompatible CFDs. This theoretical sketch is hoped to help addressing general theoretical issues such as the relationship between brain and mind, on the basis of the neural localization of cognitive elements.
\end{abstract}

Keywords: cognition, Grandmother cells; localist representations; localizationism, theory of the brain, theory of the mind ; mind and brain, hierarchy

Damien N. Fernandez, PhD

Université Picardie Jules Verne (UPJV), Amiens, France

damien.fernandez@u-picardie.fr 


\section{Introduction}

Research fields in psychology and cognitive science are often highly specialized (addressing, e.g., episodic memory, verbal working memory, motion perception, visual selective attention...) and often have little interaction with each other, or mostly dual interactions (e.g. visuo-spatial attention and working memory). This 'divide and conquer' research strategy (Kosslyn, 2006) was and still is invaluable for apprehending details of various cognitive functions. It is however insufficient.

Cognitive processes are highly interdependent from each other. For every cognitive activity, many cognitive processes are necessary and have continuous and multidirectional interactions between each other -a "coupling", in the terms of dynamic system theories. This is true at a mature stage of adult individuals' cognitive systems, and even more during the ontogenetic development. In addition, distinct functions are often based on some similar or identical psychological and / or neural mechanisms. For example, there are significant conceptual overlaps between constructs such as supervisory attentional system (Shallice, 1982), executive attentional network (Posner \& Rothbart, 2007), executive attention (Engle, 2002), executive functions (Miller \& Wallis, 2009), cognitive control (O'Reilly, Herd, \& Pauli, 2010), working memory (Baddeley \& Hich, 1974), etc.; and each of these functions is furthermore inextricably tied with other cognitive functions (vision, motor control, semantic memory...). The neural underpinnings of the former (control functions) and the latter (perceptual and motor functions) are also both overlapping and not clearly and strictly distinguishable (e.g. Andrés, 2003). Visual selective attention is also a good example to highlight the need for theoretical integration. It is often considered a genuinely independent system (e.g. Posner \& Peterson, 1990; Corbetta \& Shulman, 2002; but see Anderson, 2011). However, spatial attention has been proposed to depend at least partly on motor systems (Rizzolatti, Riggio, Dascola, Ulmitá, 1987), while exogenous featural attention is often thought to emerge within the visual system (Desimone \& Duncan, 1995, Li, 2002). Moreover, neural structures thought to underly these alleged attentional "systems" (e.g. Corbetta \& Shulman, 2002) overlap with those of other cognitive functions (e.g. inferior parietal lobe and spatial cognition: Nachev \& Husain, 2006; frontal eye field and eye movement control: Schall, 2002). If attention is indeed a property "emerging” from the functioning of other cognitive systems, or at least has strong and constitutive interactions with these latter, then studying attention independently from these latter is probably insufficient, and might even impede the genuine advancement of our knowledge of such cognitive processes.

The credo behind the present article is that conceiving a coherent account of the whole mind-brain at a very general level -its components and general mechanisms- is necessary to understand the principles of cognitive functioning and its relation with the brain. And I claim that this is true for each cognitive function considered separately, and above all for "attention". Considering conjointly the whole and its parts refers to a dialectical mode of reasoning, which goes back at least to Marx and Engels (Engels, 1952; Sève, 1998), and shares strong affinities with dynamical system approaches (Spivey \& Dale, 2006; cf. Sève \& Guespin-Michel, 2005).

Considering the mind both as a whole and as a group of parts is not sufficient, yet. It is also necessary to address, at a fine grain of mechanisms, the neural underpinnings of cognition along with their cognitive counterparts. This point is particularly important : (a) because the physical development of the brain is obviously a factor and a constraint on the development of the mind ; (b) because receiprocally, the developpement of psychological functions is a factor and a constraint on the development of the mind, whose importance grows increasingly during ontogenic development. Further, this shared account of physical 
and psychological dimensions must be thought in the light of ontogenetic development. Indeed, both the mind-brain relationships and their development through time are invaluable clues to gain insight into cognition (D'Souza \& Karmiloff-Smith, 2016). Development, for instance, strongly constrains the possible cognitive and cerebral mechanisms (Sirois et al. 2008), and can also provide some light on theoretical problems (e.g. the grounding problem: Harnad, 1990).

In summary, this article is an attempt to answer to a strong call for grand unified theories of cognition, which, although old (Newell, 1973, 1990) is still highly relevant (Barsalou, 2009; Gigerenzer, 2009, 2010; Stevens, 2000). Above the practical usefulness of such a theory of cognition to study cognitive functions, it has also an epistemological value in its own. This article aims to sketch the broad outlines of such a conceptual model of cognition in general, which addresses various cognitive processes and functions together and coherently, on the basis of neuroscientific evidence. The model's architecture and dynamics are addressed consecutively, followed by its relation with other models and its expected contribution. Finally, an account of "selective attention" is presented as a paradigmatic example.

The dynamics of the model is necessary to understand its architecture. The architecture of the model is necessary to understand its dynamics. Therefore, the author is facing a insoluble dilemna. I choosed to present one point after the other (i.e. dynamics after architecture), but I ask the reader to keep this point in mind. The best way to apprehend the proposed theorization would probably be to read the theoretical framework twice.

\section{Theoretical framework: a dynamical network view on cognition}

\subsection{A general architecture of the mind-brain}

The present model can be summarized in a general sentence as follows: Cognition can be considered as the result of the dynamical activation of a large set of Cognitive Feature Detectors (CFD), in a network organized along a bi-hierarchical structure connected with the environment at the "bottom" through effectors and sensors. This outline is elaborated below.

\subsubsection{Neural network and Cognitive Features}

Provided there is a link between brain and mind that can be theorized efficiently, one has to know and understand the functional architecture of the brain network to understand cognition. The fact that there is a link between mind and brain is roughly consensual, in scientific psychology, although the possibility that this link can be theorized efficiently is a bit less self-evident. The present essay proposes a schematic and theoretically-oriented description of the brain. This proposition is largely indebt to Fuster's (2006) cognit model insights, but presents some critical differences. One central claim of this article is that the semantic nature of the network's fundamental elements is intimately related to the network's structure, and thus, that both the elementary units and the global structure should be apprehended conjointly and dialectically.

\subsubsection{Cognitive functions}

To begin with, cognitive functions seem not to be the right analytical unit to address mind or brain issues. To link the mind and the brain, one should probably first stop trying to 
localize cognitive functions in the brain. As Fuster (2003, p. ix) put it: "[C]ognitive functions can be methodologically separated at the psychological level. It does not follow, however, that they have separate neural substrates, although this is precisely the unfounded assumption that spilled into cortical neuroscience". One might even claim that, at the psychological level, studying functions, and particularly studying functions separately from one another, is an inadequate strategy. The cerebral architecture, whose unit is the neuron, or at best the minicolumn (Mountcastle, 1997), cannot be described in terms referring to cognitive functions. Indeed, it is often claimed that any cognitive function could at best be localized at a neural network level (Posner \& Rothbart, 2007). However, it is not possible to provide a mechanistic account of a cognitive function without adressing its components, their operations and organisation. As Bechtel put it: "A mechanism is a structure performing a function in virtue of its component parts, component operations, and their organization. The orchestrated functioning of the mechanism is responsible for one or more phenomena" (Bechtel, 2006, p. 26). So, to understand how the brain "produces" cognition, provided it is possible, one have to explain cognition in the terms of the functional units of the brain. As a consequence, the attempt to localize functions is likely doomed to fail (cf., e.g., Uttal, 2001). At best, cognitive functions may only be apprehended at the level of the dynamical activation of the large parts of a cognitive-neural network embedded in a brain-world system through a perception-action cycle. Fuster (2003) defended a similar thesis when he claimed that "at any given time, a cognitive function has the distribution of the active cognits on which the function is operating" (p. 15). If cognitive function turns out not to be the ideal conceptual tool -the unit of analysis- what construct should we use instead? The answer proposed in this model rests on a rather new (although not without history) theoretical construct, labeled cognitive feature detector (CFD). One strong hypothesis of the present model is that the brain network can be described as made of neurons (or small neuronal sets) coding for CFDs in a localist way.

\subsubsection{Cognitive features: principles}

\subsection{Cognitive "bricks"}

"Cognitive features Detectors" (CFDs) are a fundamental construct in the present work. They can be thought of as nodes in a connectionist network, but they are not considered "sub-symbolic" as usually in distributed models, but they rather have a genuine semantic value -although minimalist. CFDs are the smallest and simplest atoms of information in the cognitive system, the fundamental elements. One historical root of the concept of CFDs is obviously in Hubel and Wiesel (1968)'s feature detector, that is, a neuron specifically activated when a given perceptual feature is presented in its receptive field (cf. Gross, 2002 for historical roots of the concept). CFDs, however, are not only perceptual, but can be of any kind (e.g. perceptual, executive, conceptual, etc.), they hypothetically pertain to every cognitive function, and they code for real-world entities only indirectly, as argued below.

\subsection{Categorization tag}

The first and most important defining aspect of CFDs is that they have a categorical value. They tend to have a maximal response for a given feature (either a perceptual, executive, conceptual... feature). The term "categorical value" is intended to highlight that CFDs merely refer to being something or something else, for instance: "red", "right tilted bar", "a spoon", "the act of rising up", "the concept of freedom", etc. They do not in themselves contain more information than this simple "being this (or that)". Thus, they do not "represent" anything in the strong classical sense, insofar they do not themselves hold extensive information about a "concept" considered as a whole, and by themselves they hold no "meaning" in the strong sense. For instance, a CFD labeled "blood" would not contain the 
information of "being red", "being in a body", "being liquid", etc. It would only code, very minimally, for the category "blood".

Actually, the associated information and the semantic information contained in the very concept of blood, would lie in the network constituted by this CFD the CFDs to which it is connected, directly or indirectly, and in the specific pattern of connections (and particularly in the connection weights) between these CFD. For instance, the connections between the CFD for the category "blood" and semantically associated CFDs (e.g., hypothetically: color red, liquidity, injury...) would partly underlie the concept of blood. This point can evoke the classical idea that partly defines connectionism according to McClelland, Botvinick, Noelle, Plaut, Rogers, Seidenberg, \& Smith (2010): namely that "knowledge underlying cognitive activity is stored in the connections among neurons” (p. 348). The point is clearly different however, since a localist coding is postulated that proponents of PDP usually reject.

\subsection{Localizationism and "grandmother cells"}

CFDs are claimed to be coded in a localist manner in the network (cf. Bowers, 2009). Many authors in connectionism (e.g. Plaut \& McClelland, 2010) might argue that cognitive entities (e.g. representations) are not coded in a local but in a "distributed" way, and that the network units (i.e. nodes) are semantically neutral and do not code for anything individually. Indeed, Smolensky (1988) argued that: "the entities in the intuitive processor with the semantics of conscious concepts of the task domain are complex patterns of activity over many units. Each unit participates in many such patterns” (p. 6). On the other hand, he also considered that "on the side of what is represented, the conceptual level is populated by consciously accessible concepts, whereas the subconceptual level is comprised of fine-grained entities beneath the level of conscious concepts" (p. 63). So one can conclude that, at least in the the Smolensky brand connectionist approaches, fine grained features are coded locally while more abstract concepts are coded in a distributed way. In the present model, it is claimed that both "sub-conceptual" features and concepts are coded locally in the nodes of the network. The view that cognitive entities are distributed over large sets of neurons is very widespread. The success of this doctrine is, however, probably overrated, and there are many reasons to question it.

First, there clearly exists localized features in perceptual neural systems, the most consensual being those in low level perceptual and motor systems (e.g. Hubel \& Wiesel, 1968; vcf. Bowers, 2009). Yet there are no clear reasons for assuming arbitrarily qualitative change in coding type between different levels of processing (i.e. between "sub-conceptual" and "conceptual" entities). Second, Bowers (2009, 2010, 2017) argued that many networks, including deep neural networks and networks claimed to be distributed, are actually genuinely localist (e.g. McClelland \& Rumelhart, 1981; Poggio \& Bizzi, 2004). Third, localist models can sometimes describe more accurately empirical findings than connectionist models (Seidenberg \& Plaut, 2006; Bowers, 2010). Fourth, localist models might actually be more computationally efficient than distributed ones (e.g. Bowers, Vankov, Damian, \& Davis, 2014; Collins \& Jin, 2007; Mayor \& Plunkett, 2012). Therefore, looking for localist coding of cognitive entities might be a productive scientific strategy, deserving to be pursued (cf. Barlow, 1972; 2009; Grainger \& Jacobs, 1998; Gross, 2002; Roy, 2012, 2013, 2015, 2017; Thorpe, 2011, for other defenses). However, as argued here, one should probably try to localize something like CFDs rather than cognitive functions.

A comparison can be made between the construct of CFD and the disputed and infamous concept of localist representation cells (or "grandmother cells", more colloquially; cf. Bowers, 2009). According to Bowers (2010), "the defining feature of a localist representation is that it codes for one thing, and accordingly, it is possible to interpret the activation of a single unit” (p. 222, italics added). He insisted that the defining feature is not 
that "one and only one neuron codes for a given item" (p. 223). The idea of a grandmother cell could be understood as a simple extension of the complex cell theory presented by Hubel and Wiesel (1962, 1968). Indeed, a cell can respond specifically to a given feature, which is the combination of set of simpler features (coded by the neurons that project on it). This kind of nodal cells exists at many hierarchical levels, as clearly illustrated in the visual system (Riesenhubber \& Poggio, 1999).

Thus, in some sense, the present model can be categorized as a localist connectionist one. Some points must be made, however. First, CFDs do not directly refer to stimuli, or real world perceived entities, but only to other CFDs. Indeed, except sensors (i.e. CFDs transducing physical or chemical stimuli in signals in the cognitive network), all CFDs are only "composed" " of other CFDs, that is, they are defined in terms of other CFDs. Therefore, CFDs do not code directly for concepts, stimuli or things in the world but, strictly speaking, for combinations of CFDs. Second, Bowers (2009) ascribed a function of "representation" to "grandmother cells". Instead, the notion of CFD defended has a more minimalist acceptation than the notion of "representation" as usually used. Third, it must be highlighted that CFDs, contrary to symbols in the computational theory of mind, have an active functional role and truly participate to cognition. Indeed, cognition is considered, literally, as the dynamic activation of CFDs in the cognitive network. Therefore, CFDs are not "objects" manipulated by cognitive processes or systems, but are an integral part of the processes themselves, through there connections.

Some concluding remarks might finally be made about the notion of CFD. It is not assumed that every concept is represented as a CFD. A "red-head grand-mother smiling" would probably not be coded as a CFD. Indeed, as argued in the next section (2.1.1.2.4 Weighted spatiotemporal combinations), CFDs are not directly made for representing anything; they only emerge as a function of statistical regularities. Some things or events are simply not often enough encountered, or not enough attended to, to give rise to specific CFDs. It can be hypothesized that dynamical activation of the network, however, will permit, through the activation of several CFDs (i.e. red-head, plus grandmother, plus smiling, etc.) to produce effects in activation dynamics that are quite similar to the one that would be obtained by the activation of a hypothetical "smiling grand-mother" CFD (cf. Bowers, 2009 for a similar reasoning about localist representation cells). In addition, CFDs are usually quite stable but are not unchangeable. The system is completely dynamic and every CFD is potentially susceptible to change, following changes in the activation patterns on CFDs that project on it. Finally, one must write that the existence and validity of CFD is an empirical question, a very difficult one, but as such it will have to be addressed empirically.

\subsection{Weighted spatiotemporal combinations}

The rules governing activation spread in the cognitive space are classical connectionist ones (Feldman \& Ballard, 1982; Thomas \& McClelland, 2008). The most important ones are briefly restated below to make them clear in the present context, and above all, to enlighten the nature of the CFDs. The categorical value of a CFD is strictly defined through the weighted spatiotemporal combination of the other CFDs that project on it (i.e. its afferent CFDs). The firing response efficiency of a given CFD would likely be optimal when all its afferent CFDs are activated in their optimal temporal order. This optimal situation -the activation of all excitatory and none of the inhibitory afferent CFDs- is highly improbable, however. Most often, only a part of excitatory afferent CFD would be activated (and some of the inhibitory ones). In this situation, a CFD might still be activated, as soon as the weighted sum of its afferent CFDs exceeds a given threshold at a given time (the notion of a threshold

1By the notion of composition, I refer to the way in which a CFD is defined by the CFDs that project on it, that "compose" it. Is also write that these CFDs "combine" on it. 
is not necessary, however, and a different response function is possible). Among all effectively existing co-activations of CFDs, the one that would activate the target CFD the most efficiently corresponds to a prototypical value. During perception, a CFD is likely to be activated for a prototypical stimulus (e.g. a dog for an animal CFD; Rosch, 1978). The influences of afferent CFDs are not all equal in intensity: some CFDs have a stronger influence on their targets, and their connection weight is higher. To take an imaginary example: the nose is likely to be more critical for perceiving a human face than the Adam's apple or a moustache. The weights of the nose-to-face (CFDs) connection would therefore probably be stronger than those connecting the Adam's apple or moustache (CFD) to the face (CFD). The rules that make connection weights change are simply Hebb-like rules, on the basis of statistical contingencies of afferent and target CFDs (co-)activations. The more regularly co-activated are the afferent and target CFDs, the more their connection strength will tend to increase.

\subsection{Some consequences}

Some important consequences stem from these principles. First, it is well known that connectionist networks present a pattern completion property (McClelland \& Rogers, 2003; Trappenberg, 2009). Thus, CFDs can generalize for unknown elements of a category or err in categorizing. Second, CFDs are not function-specific. Indeed, as a part of a complex network, a given CFD can be part of many patterns of activation. Therefore, it can participate to various cognitive processes. For instance, a CFD "color blue at location XY" should obviously be activated for many visual stimuli. At the neural level, the posterior parietal cortex might be involved in space perception (Andersen, Snyder, Bradley, \& Xing, 1997), but also in sensorimotor integration (Culham, Cavin-Pratesi, \& Singham, 2006), in spatial attention (Behrman, Gene, \& Shomstein, 2004; Posner \& Petersen, 1990), and even in episodic memory retrieval (Wagner, Shannon, Kahn, \& Bruckner, 2005) or premotor control (Buneo \& Andersen, 2002). This might be due to the fact that different cognitive processes involve the same CFDs (e.g. coding for some spatial localization) to be activated, though in different ways (i.e. the whole dynamical activation's pattern is different). Analyzing the tasks in which individuals are engaged, and the processes associated, through the concept of CFD might help understanding and predicting which parts of the cognitive space (and thus of the brain) would be activated at what time.

\subsection{2 "Cognitive space" / Representational map}

The CFDs are fundamental elements in the present model. However, 1) they only take their meaning from their position in the neural / cognitive network; 2) their genuine cognitive nature only emerges through the dynamic activation of the network. Therefore, it is necessary to describe the general principles of the architecture and activation dynamics of the network (cognitive and neural network). First, the notion of "cognitive space" is presented and described, along its two fundamental meaningful dimensions: sensorimotor (plus "modalities") and hierarchical dimensions.

\subsubsection{Cognitive - neural isomorphism}

Within the present conceptualization, the constructs of CFDs and neurons are directly linked. Indeed, each CFD can be directly attributed to a neuron or a small assembly of neurons in the brain (as initially thought by Hebb, 1949). That is, the strong hypothesis is that there is a direct isomorphism between CFDs and neurons, between cognitive and neural networks. Put differently: the highly complex organization of the neural system can be understood in relation to the organization of the CFDs in the cognitive system (and vice- 
versa). Each neuron can be assigned a CFD and the structural organization of neurons is isomorphic with the global architecture of CFDs in the cognitive system. This isomorphism will allow us to mechanistically account for cognition and mind-brain relations. It must be stressed that this isomorphism could only be found at the specific levels defined, that is; neurons or small groups of neurons on the neural side -and not 'networks'- , and CFDs on the psychological side -and not 'concepts', 'representations' or 'functions'.

\subsubsection{General architecture}

Given the hypothesized isomorphism between neural and cognitive networks, it is proposed to refer to the neural architecture as a global cognitive space (represented in figure 1 , top panel) when emphasis is put on cognition rather than brain. Cognitive space is roughly similar to what Fuster (2004) called 'representational map'. Every CFD should be localized in this cognitive space. This architecture is important for two reasons at least. On the one hand, the position of a CFD in this cognitive space (along with its precise pattern of connections) determines its characteristics (i.e. its semantic value). Indeed, the pattern of connections, and thus the location in the network, of a CFD, is (mechanistically speaking) the only way by which it acquires a semantic value. On the other hand, the general architecture determines the CFDs' dynamics of activation, thus cognition per se. This is why architecture is strongly emphasized. The two most important characteristics of cognitive space, also stressed by Fuster (2006), will first be described. First, the cognitive space is roughly divided in two (highly connected but conceptually distinguishable) parts: one motor-executive and one perceptual-perceptive. Second, both are organized mostly hierarchically, allowing for more and more complex-abstract CFDs as one moves higher.

\subsubsection{1 sensorimotor dimension}

First, the whole cognitive - neural system can be divided in two distinct parts: one "perceptive" and one "executive". These two words were chosen because they both refer at the same time to the two fundamental cognitive functions (perception, and action or motor function) and at higher cognitive functions (intelligence, and executive control) ${ }^{2}$. Indeed, on the one hand, motor and executive units are thought to lie respectively on the bottom and top ends of an executive part or axis. On the other hand, perceptual units and abstract (semantic, episodic...) units are thought to lie respectively on the bottom and top ends of a perceptive system or axis. The two parts, executive and perceptive, are rather neatly separated. Additionnally, in each part, a CFD can be more or less close to the frontier between executive and perceptive, that is, more or less directly connected with CFDs of the other part.

\subsection{Hierarchical dimension}

The second major dimension is hierarchy. In this context, hierarchy refers to the increasing complexity or abstractness of CFDs along this dimension. This increasing complexity - abstractness of CFDs at higher and higher levels is considered to be the result of the iterative combinations of lower level CFDs, progressively constructed during ontogenic development, through experience. This principle exists for both parts (perceptive and executive).

Perceptive and executive systems are organized roughly hierarchically in the brain, in such a way that CFDs at a given level are usually combined together at the next (higher) level, creating more complex - abstract CFDs. Complexity - abstractness is driven by the pattern of convergence of neurons, from lower levels (closer from sensors or effectors) to higher levels (i.e. farther from sensors or effectors). This hierarchy can be (at least theoretically)

2The term executive, in the present sense, is due to Fuster (e.g. 2006).

Fernandez (2020) CFD model 


\section{Executive Cognits Perceptual Cognits}

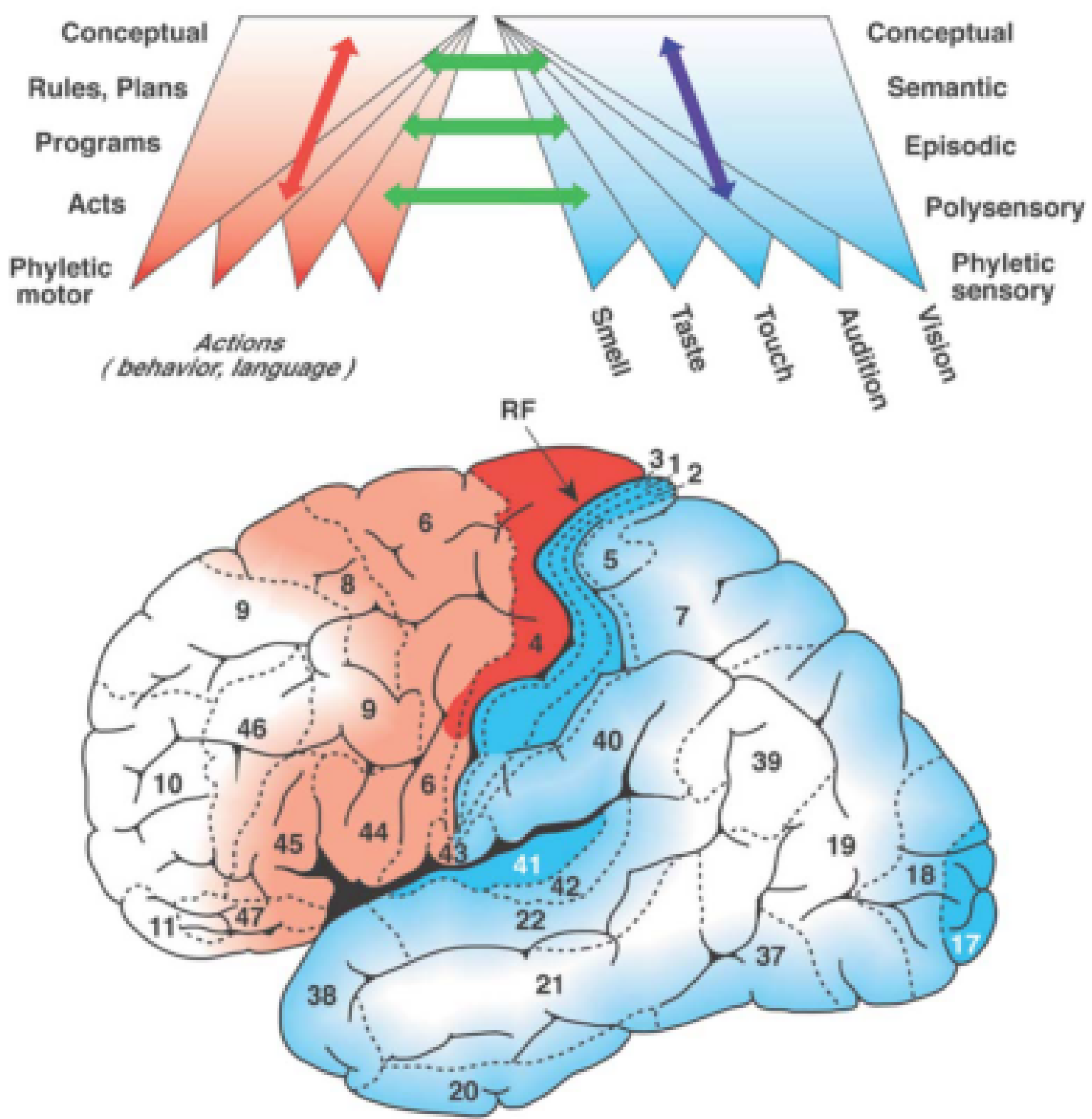

Illustration 1: This figure depicts, on top, the cognitive space, that is, the theoretical architecture of the mind-brain, as presented in Fuster's cognit model (Fuster, 2006). The perceptive part is on the right (in blue), the executive part is on the left (in red). From the bottom up, following the hierarchical axis, cognitive features are more and more complex and abstract. The order and location of CFDs" kinds (i.e. from "phylectic" to "conceptual" ...) is not necessarily the same as in the present framework; notably, episodic, semantic and conceptual CFDs are supposed to be roughly at the same hierarchical level, although at different location in the cognitive space. The approximate cerebral localization of the CFDs (and of the cognits, in Fuster's conceptualization) are represented in the lower panel. Reprinted with permission from Fuster (2006).

decomposed in multiple stages of integration, so that the integration is progressive and leads to gradually more complex - abstract CFDs.

Iterative hierarchical combination of CFDs of growing complexity is probably very powerful computationally, obviously more than the mere direct combination of simple CFDs. 
Riesenhubber and Poggio (2000) highlighted some computational advantages of postulating a hierarchical organization of the visual system:

"The gradual and parallel increase of feature complexity and receptive field size, as found in the visual system, is crucial in avoiding a combinatorial explosion of the number of units in the system on one hand or insufficient discriminatory ability on the other hand. Although the invariance range is low at lower levels, thus requiring many cells to cover the required range of scales and positions, only a small set of simple features must be represented. Conversely, in higher layers, where neurons are tuned to a greater number of more complex features, neurons show a greater degree of invariance, thus requiring fewer cells tuned to the same feature at different positions and scales" (p. 1201).

This hierarchical integration principle, however, is much more than an "engineer's trick". There is much suggestive evidence in favor of hierarchical organization in many cognitive domains and neural structures (see below, section 2.1.2.2.4 Detailed description of the two parts), even if this organization is not yet clear and consensual for every cognitive domain.

To be clear, the point is not to claim that all the brain is strictly hierarchical and that no bypass between levels, lateral connections, or even top-down connections exist. The hierarchical combination pattern is a general principle and all of these kinds of connections are recognized to abound. First, many divergent pathways exist, which allow segregated processes (e.g. modality-specific pathways within the visual system: colors, forms, faces...). Second, connections between CFDs can bypass levels. Third, lateral connections are also postulated, as a possible way to account for lateral competition for instance (cf. Section 2.2.2.1.5 Horizontal, inhibitory connections). Fourth, it is even claimed that most "top-down" connections are reduplicated by reciprocal bottom-up connections, as defended by Rolls (2008). This additional set of combination kinds can be referred to as "heterarchical" (Bernston \& Cacioppo, 2000; Fuster, 2003). It does not contradict the general principle of hierarchy. Indeed, the claim is that there is a hierarchical iterative combination pattern, which is necessary to account for CFDs of various abstractness or complexity levels -that is, these (abstract and complex) CFDs could not appear in a mind without such a hierarchical pattern.

2.1.2.2.2.1 Precisions on complexity / abstractness

Combination can lead to increased abstraction or complexity in different ways. This is why the point is kept quite vague and general. Indeed, combination between CFDs can give rise to a more complex CFD, defined for instance as the structural combination of several elements (think, e.g., of a triangle, as composed of three segments correctly arranged). The combination might also refer to a taxonomic level (generalization; Dove, 2016), such as, for instance, when the combination of CFDs for various known cat breeds or types would give rise to a generic cat CFD.

\subsection{CFDs local organization}

2.1.2.2.3 Cognitive Maps

Throughout the network, CFDs are organized in cognitive maps. A cognitive map is a bi-dimensional cortical representation of a perceptive or executive space, in which nearby items in the original space (e.g. visual space; but spaces can be of a more abstract kind, e.g. semantic) are represented by nearby neurons on the cortical sheet. As argued by Graziano (2006; 2010) for cortical representation of actions in the motor cortex, the represented space (e.g. visual space, grasping space, more abstract cognitive space...) might be organized along more than two dimensions. Some maps (e.g. in motor cortex) can therefore present much less 
continuity than others (e.g. in V1). The CFDs' organization in multiple cognitive maps is supposed to be a consequence of the combination and learning principles of the network (see below, section 2.2.3.2Map construction principles). Topographic maps are a major organizational pattern in the human cerebral cortex (Silver \& Kastner, 2009; Wandell, Dumoulin, \& Brouwer, 2007), which extends to other perceptive modalities (e.g. olfactory; Murthy, 2011) and motor systems (e.g. Graziano \& Gross, 1998), and have also been hypothesized for higher level cognitive functions (e.g. Franconeri, Alvarez \& Cavanagh, 2013; Thievergie \& Markus, 2007).

2.1.2.2.3.2 Maps for perceptive and executive modalities

At the lowest hierarchical levels, the perceptive part of the system is not homogeneous, but rather roughly organized according to perceptual modalities (e.g. vision, audition, etc.) and sub-modalities (e.g. perception of color, movement, pitch, etc.). These modalities, spatially organized in maps, are related to various physical or chemical stimuli, and have specific spatial organizations, in the body and in the brain. The cognitive maps, at higher levels, are partly determined by these "initial" perceptual and motor categories, thanks to iterative combination and convergence patterns. Perceptive maps are postulated even at higher hierarchical levels, perhaps up to the highest ones, such as for working memory (Sala, Rämä \& Courtney, 2003), and one can imagine up to semantic maps (Ritter \& Kohonen, 1989, for a computational account).

Similarly, on the executive side, some network parts are rather segregated, for instance those coding for different body members (such as hand or leg). But at higher levels, this spatially-based segregation is likely to be overcome. Indeed, actions may often imply combined movements of distinct members (Rizzolatti, 2001). As a result, organization in maps can be related to more abstract principles, such as behavioral repertoire, for example (Graziano, 2006, 2010, 2016).

\subsection{Detailed description of the two parts}

It is simpler to understand the hierarchical organization of the brain network when executive and perceptive parts are first considered separately. This is not to say that these parts function separately or are completely independent: on the contrary, they are claimed to be highly connected, both structurally and functionally, as suggested by notion of perceptionaction cycle (Fuster, 2006; Uexküll, 1926). Indeed, these two parts are at least partly interconnected, even from the lowest levels (e.g. reflex-like connections). The distinction is still very important, however. One obvious reason of this is that the perceptive and executive neural systems are structurally clearly separated, as is clear in Figure 1 (lower panel). So, the two domains are considered separately at least for a descriptive purpose. The distinction between perceptive and executive parts of the network is most evident at the lowest levels among sensors and effectors. A similar hierarchy is built on each of these domains (perceptive and executive).

2.1.2.2.1 Perceptive part

Hierarchy is a key feature of the brain organization (cf. Fuster, 1997, 2006, 2009). Probably the best known hierarchical model is for the ventral visual system (cf. Figure 2; Ahissar, Nahum, Nelken \& Hochstein, 2008; Hedgé \& Felleman, 2007; Hubel \& Wiesel, 1968; Riesenhuber \& Poggio, 2000; Serre, Oliva \& Poggio, 2007; Markov et al., 2014; Vezoli, Gariel, Markov, Anderson, Douglas, Martin, Knoblauch, \& Kennedy, 2010). It is usually accepted that the visual system is roughly divided in two pathways, the ventral What? pathway coding for object's identities, and the dorsal Where? / How? pathway coding for spatial localization and/or visually guided action (Mishkin \& Ungerleider, 1982; Goodale \& 
Milner, 1992), although the details are probably more complex (Binkokski \& Buxbaum, 2012; Pisella, Binkosfki, Lasek, Toni, \& Rossetti, 2006; Rizzolatti \& Matelli, 2003).

\subsection{Hierarchy in the ventral visual pathways}

Concerning the ventral pathway, Hubel \& Wiesel (1968) evidenced that in the cat's primary visual cortex some neurons code for spots of light, thanks to the spatially organized combination of neurons coding for presence (vs. absence) of light stimulus in their receptive field. Some neurons code for oriented bars, as neurons coding for spots whose receptive fields are aligned project on them. In the ventral visual pathway, neurons code for progressively more complex features from simple spots of light, to On/Off patterns, to oriented bars, etc., thanks to the anatomical wiring allowing hierarchical iterative combinations of spatially organized neurons. These authors proposed the "possibly over-simplified concept of a hierarchical system dependent on anatomical wiring, in which geniculate cells with concentric fields converge on simple cortical cells, simple cells in turn converge upon complex cells, and complex on hypercomplex" (p. 217).

This principle might easily be extended to more complex CFDs (Risenhuber \& Poggio, 1999, cf. Bowers, 2009). For a hypothetical example in the visual system, oriented lines coded at one level might be combined at the next level to code for a form such as a triangle. In the perceptive domain, along an occipito-temporal axis, areas code for more and more complex CFDs (Tanaka, 1997; Lehky \& Tanaka, 2016), from simple perceptive CFDs (oriented lines, colors, simple motions...) at the bottom, to complex unimodal perceptive CFDs (e.g. faces, visual images of complex objects...) to multimodal perceptive CFDs (e.g. a cat considered according to the combination of its visual image, sound and texture, etc.) to more abstract CFDs (e.g. the concept of "parsimony"), or to "episodic" (mnemonic) CFDs.

And indeed, neurons responding quite selectively for very complex visual features, such as complex geometrical features (Tanaka, 1996), specific faces (Perrett, Rolls, \& Caan, 1982; Tsao, Freiwald, Tootell, Livingston, 2006) famous persons (Quian Quiroga, Reddy, Kreiman, Koch, Fried, 2005) or monuments (Quian Quiroga, Reddy, Kreiman, Koch, Fried, 2007) were found in the temporal lobe (cf. Quian Quiroga, 2012, and Roy, 2013).

\subsection{Multisensory integration for action and for identification}

One might draw a parallel with the research field about multisensory integration, and explore the link between usually separated fields of research (i.e. space perception, semantic and episodic memory and multisensory integration). "Multisensory integration" refers to "the neural processes that are involved in synthesizing information from cross-modal stimuli" (Stein \& Stanford, 2008). Multisensory integration might arise at several levels of the perceptive hierarchy (e.g. superior colliculus, primary auditory and visual cortices, ventral and lateral intra-parietal areas; e.g. Koelewijn, Bronkhorst \& Theeuwes, 2010, Driver \& Noesselt, 2008, Ghazanfar \& Schroeder, 2006 for reviews). Here, "multisensory integration" is simply considered as a combination of CFDs from several different perceptive (sub-)modalities. 


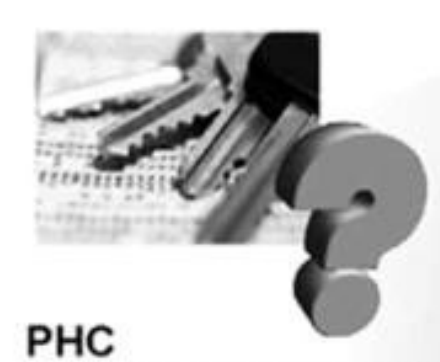

PHC

current visual task instructions

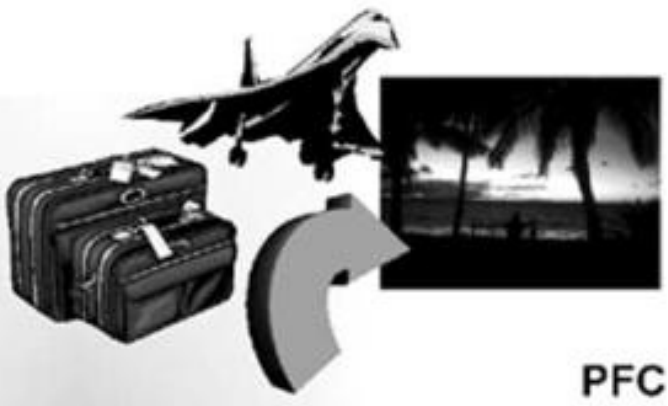

behavior, planning

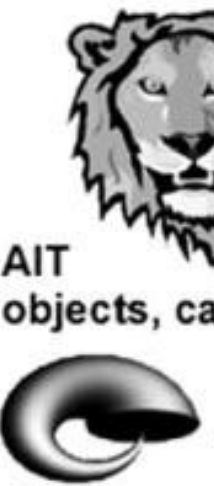

PIT
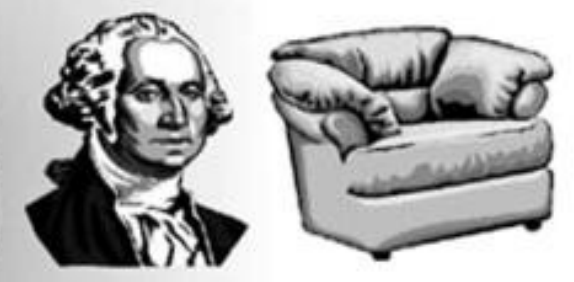

AIT

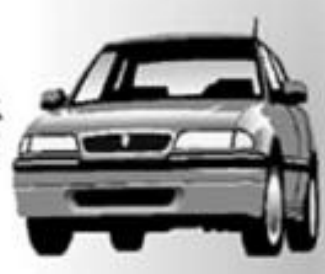

ategories

complex shapes, object components

V4
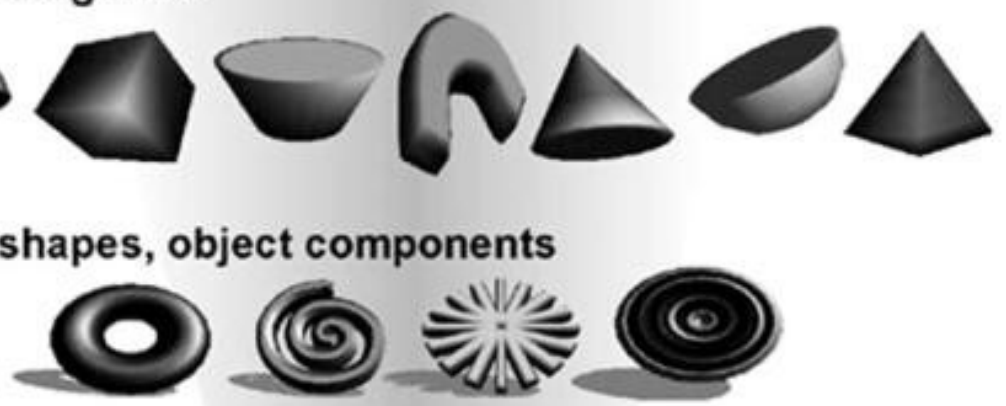

complex geometric patterns

\section{V2}

figure/ground, illusory contours

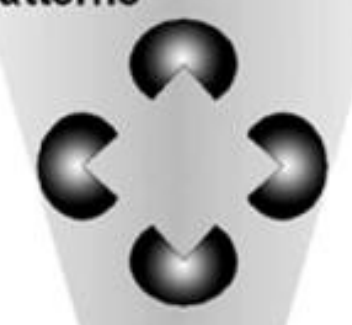

V1

line/edge orientation

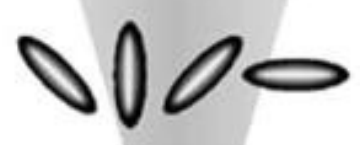

\section{LGN}

\section{luminance contrast + ?}

\section{Retina}

luminance contrast

Illustration 2: The figure 2 depicts the hierarchy of CFDs in the ocipito-temporal ventral visual stream. PHC: parahippocampal cortex; PFC: prefrontal cortex; AIT: anterior part of the infero-temporal cortex; PIT: posterior part of the inferotemporal cortex; V1, V2, V4: Visual areas 1, 2, 4; LGN: lateral geniculate nucleus. Reprinted with permission from VanRullen (2003). 


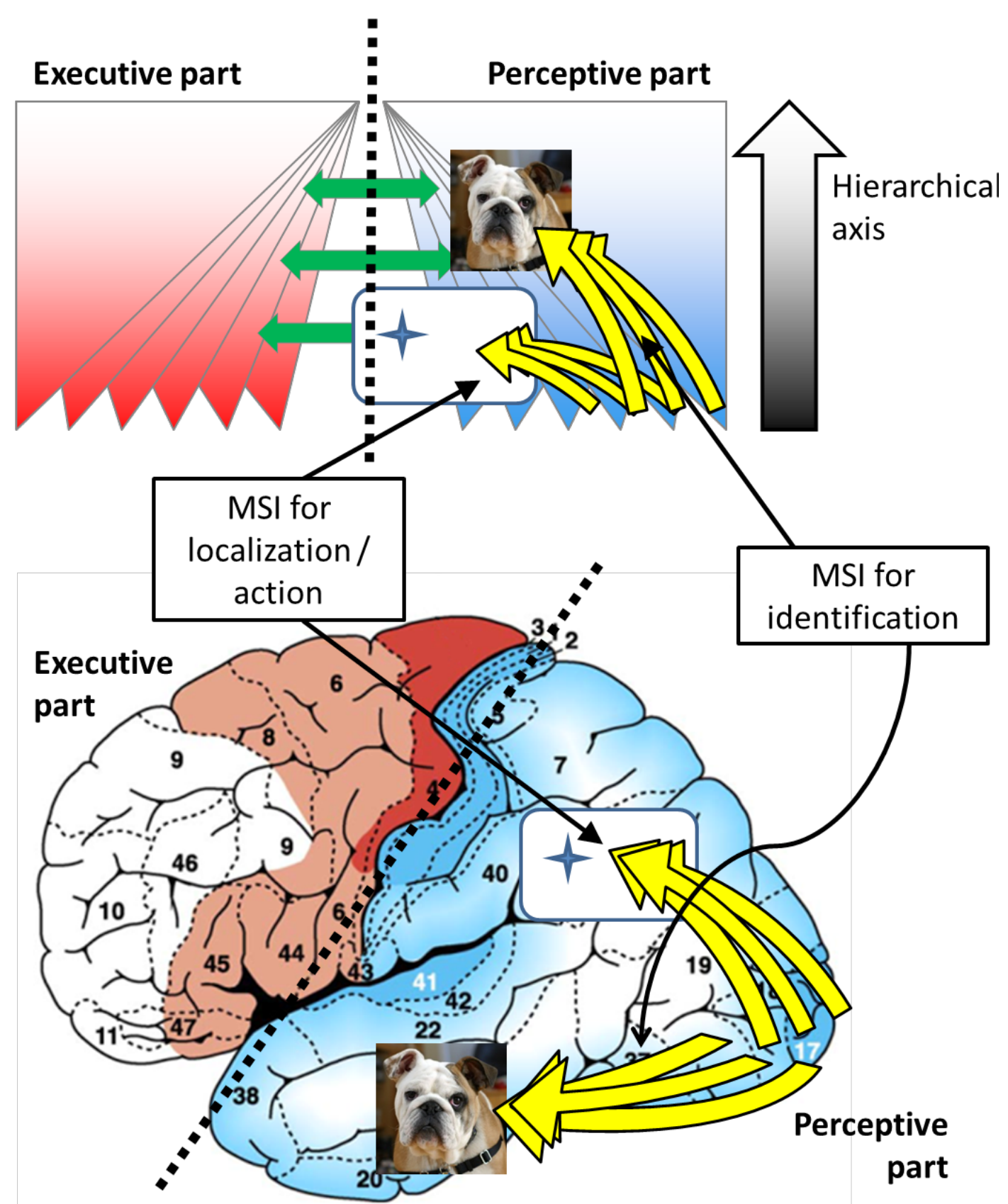

Illustration 3: This figure schematically represents the localization in the cognitive space (top panel) of two kinds of multisensory integration (MSI; Stein \& Stanford, 2008), namely for object identification and for localization / action. MSI for identification involves high level perceptive CFDs, located higher in the cognitive space. MSI for localization action involves lower level perceptive CFDs. In addition, these CFDs are more tightly related with executive CFDs thus closer to them. The approximate cerebral localizations of the pathways associated with MSI for identification and localization action are schematically represented in the lower panel. On the basis of Fuster (2006). 
Such multisensory integration might occur either in dorsal or ventral visual pathways. This anatomical distinction would reflect the dichotomy between (a) multisensory integration for localization (and / or action) purpose and (b) multisensory integration for identification purpose proposed by Stein and Stanford (2008, see also Spence, 2013). Stein and Stanford (2008) suggested that the principles of multisensory integration differ between some multisensory integration structures and others (e.g. superior colliculus vs. superior temporal sulcus). According to these authors, spatial and temporal congruency, on the one hand, were highly relevant for superior colliculus, a structure involved in orienting attention and eyes toward relevant or salient spatial regions. On the other hand, these characteristics (localization and timing) are less relevant than other semantic properties for superior temporal sulcus, where more abstract / complex (e.g. the sound and vision of tearing a piece of paper with two hands) characteristics need to be congruent to produce a multisensory activity enhancement (Barraclough, Xiao, Baker, Oram \& Perrett, 2005).

In the present model, this difference would similarly be accounted for by the nature and characteristics of the CFDs that are combining in the multisensory integration CFD. The multisensory integration for spatial (and / or action) purpose would take place at a low level of the perceptive part and close to -on a pathway directed toward- the executive part, while the multisensory integration for identification purpose would stand higher in the perceptive part (see Figure 2).

So, in the dorsal pathway(s), it is claimed that CFDs can selectively code for simple spatial localization by combining different kinds of CFDs on the basis of their receptive fields' localitions or spatial configuration, independently of other featural characteristics. Spatial integration might occur for instance in superior colliculus (Stein, Jiang \& Stanford, 2004) or lateral intra-parietal area (Andersen, Snyder, Bradley \& Xing, 1997). In superior colliculus, some neurons (CFDs) receive projections from several perceptive modalities (visual, auditive and somatosensory; Stein et al., 2004), on the basis of the receptive field's location. Such spatial CFDs in the superior colliculus provide a spatial code for visually- or auditivelydriven attention, or gaze-orienting (Stein \& Stanford, 2008). Similar mechanisms occur in posterior parietal cortex, where precise spatial information is largely preserved while nonspatial information is largely lost through the connection pattern (Andersen et al., 1997).

Concerning other spatial characteristics, in particular affordances for actions (e.g., grasping), they probably involve both ventral and dorsal pathways and shape-related CFDs.

Importantly, spatial CFDs are close to low- and mid-level executive CFDs, related with motor actions, and they probably receive feedback afferences from them. This would account for the "sensorimotor transformation" function ascribed to cerebral structures of the dorsal pathway (e.g., Buneo \& Andersen, 2006). For instance, some visuospatial maps might receive feedback from eye-movement control executive CFDs, allowing for visuo-attentional processes (Iacoboni, 2000). Other kinds of executive feedbacks are expected on different perceptivo-motor maps of the dorsal pathway, which might account for known influences of various kinds of motor preparation on visuo-attentional orienting (Rizzolatti \& Craighero, 2010; Tipper, Lortie, \& Baylis, 1992).

Conversely, in the ventral pathway, CFDs can code for non-spatial features or object identity with some spatial invariance, by combining perceptually similar CFDs with distinct receptive fields. Multisensory integration for identification is thus supposed to rather take place in the occipito-temporal structures (e.g. superior temporal sulcus). For instance, CFDs coding for complex characteristics (e.g. the moo of a cow and the visual percept of a cow, etc.) might combine on a multisensory CFD for a cow. This CFD would be more abstract and general, possibly semantic. This semantic multisensory integration is identical to the general iterative hierarchical combination principle for semantic CFDs (cf. section 2.1.2.2.4.1.4 CFDs for multimodal perception and semantic memory). 
In summary, multisensory integration for identification and for localization can be subsumed under the proposed hierarchical iterative combination principle, and their difference can be accounted for by considering the kinds of CFDs they respectively combine and the way they do so. The multisensory integration for spatial (and / or action) is supposed to take place at a low level of the perceptive part and close to the executive part, while the multisensory integration for identification purpose would stand higher and in the perceptive part (see Figure 2).

\subsection{3 Hierarchy for other perceptual CFDs}

The visual system is probably the best known perceptive system, but it is not the only one to be organized hierarchically. For instance, the auditory cortex is also considered to be hierarchical (e.g., Kaas, Hackett \& Tramo, 1999; Leaver \& Rauscheker, 2010; Okada, Rong, Venezia, Matchin, Hsieh, Saberi, Serences \& Hickok, 2010; Sharpee, Atencio, Schreiner, 2011; Wessinger, VanMeter, Tian, Van Lare, Pekar, Rasuchecker, 2001), as well as the neural network underlying haptic object processing (James, Kim \& Fisher, 2007). These modalities are also proposed to be roughly divided in dorsal and ventral streams (Dijkerman \& De Haan, 2007; Rauschecker \& Scott, 2009; Sedda \& Scarpina, 2012).

\subsection{CFDs for multimodal perception and semantic memory}

A hierarchical organization would likely be accepted for low level perceptive systems, but what about more abstract / complex CFDs? The case of semantic memory is evaluated first. Usually, semantic knowledge refers to some general knowledge about objects, persons, ideas, etc., which is independent of the context in which it was learned, and of particular details, and which does not involve the self. Here, the claim is that semantic CFDs are no more than some kind of high-level (perceptive or executive) CFDs. Iterative and multimodal combination allows to extract abstract categories devoid of the specific characteristics associated with the individuals of the category (e.g. the red color of my bicycle or the particular form of its handlebars, vs. the abstract idea of a bike). These CFDs are semantic, but are also executive or perceptive. For instance, when a dog is perceived and recognized as a dog, a dog CFD is claimed to be activated; and it is hypothesized that the very same CFD is activated when one think to or imagine a dog, in an abstract way (I.e. not a particular dog as it was perceived at a particular time and place).

This approach rejects any strong perceptive / semantic dichotomy. Usually, semantic is strictly restricted to high level knowledge, and is strictly separated from the perceptual ${ }^{3}$. However, no clear and unequivocal distinction between semantic and perceptive elements can be made. Both categorize other elements and both are abstract to some extent. Indeed, even two simple stimuli can be physically or perceptually very different (and they are almost always at least slightly different) and still elicit a similar response: thus even a low-level perceptual feature is abstract in a sense that is not trivial. The difference between perceptual and semantic CFDs is at best quantitative. It is a difference in the degree of abstraction of the categorized elements - perceptual elements being considered more concrete a priori- and the frontier can only be traced arbitrarily.

Besides this theoretical consideration that all CFDs could be considered abstract to some extent ${ }^{4}$, many empirical evidence suggest that semantic information could indeed be coded in individual CFDs, thanks to iterative and multimodal combination. In the medial

3Actually, even in the grounding cognition approach, the distinction between semantic and perceptivo-motor elements is emphasized, since the former are claimed to be grounded on the latter. This necessitates a conceptual a radical and un-dialectical distinction between the two concepts (Fernandez, upcoming). 
temporal lobe, many "concept cells" were found in the human brain, particularly in medial temporal lobe, selectively responding to specific individuals, landmarks, animals, objects (reviewed in Quian Quiroga, 2012; Roy, 2015; Suthana \& Fried, 2012). Several characteristics of these neurons are relevant here.

Interestingly, some neurons responded independently of the stimulus modality (i.e. visual picture, pronounced or written name; Quian Quiroga, Kraskov, Koch, \& Fried, 2009), or despite large variations in size, position, or viewing angle, of the pictures presented (Quian Quiroga, Reddy, Kreiman, Koch, \& Fried, 2005). Quian Quiroga, Mukamel, Isham, Malach, and Fried (2008) evidenced that this kind of neurons responded only when the stimuli were consciously recognized, suggesting a fundamental role in conscious categorization. Using fMRI, Leaver and Rauscheker (2010) also found evidence for coding of auditory object category (e.g. cat, trombone...), at the top of a hierarchically organized auditory-processing stream (see also Tian, Reser, Durham, Kustov, Rauschecker, 2001, for single cell recordings). In sum, there's some evidence suggesting that amodal and abstract CFDs are plausible at least in the medial temporal lobe.

Kreiman, Koch, and Fried (2000) also found neurons coding for larger categories, such as faces, natural scenes and houses, famous people and animals. Lin, Chen, Kuang, Wang and Tsien (2007) evidenced that some hippocampal cells responded to the abstract concept of nest, independently of several variations (e.g. location, form, size, and so on). Remarkably, these neurons did not respond to the same object rendered useless (by blocking the entrance by a glass floor), which further suggested that they truly coded for the concept of nest rather than for other less abstract characteristics (e.g. perceptive). Yoshida and Mori (2007) "showed that many cortical neurons [in the dorsoposterior part of rat anterior piriform cortex] are tuned selectively to either a single category or a specific combination of distinct categories" (p. 9105; cf. Roy, 2015).

In addition to coding for semantic categories, some neurons can display the expected mnesic properties. Kreiman, Koch, and Fried (2000) found neurons that were active when patients imagined stimuli. Interestingly, a relatively large number of them was also active during visual perception of the same stimuli. This involvement in both perception and memory is coherent with the hypothesis of CFDs.

The medial temporal lobe as well as the anterior temporal lobe are both at the apex of the perceptual neural system hierarchy. For medical reasons, most of the human single-cell data concern the medial temporal lobe (Rey et al., 2015). On the other hand, there is a wealth of imaging and neuropsychological evidence involving the anterior temporal lobe in semantic memory (e.g. Lambon Ralph, Sage, Jones, \& Mayberry, 2010). In particular, the anterior temporal pole has been proposed to underlie a kind of semantic hub (Lambon Ralph et al., 2010; Patterson, Nestor, \& Rogers, 2007; Simmons \& Martin, 2009), allowing to code for semantic knowledge, although possibly or partly biased toward social and personally relevant stimuli (Wong \& Gallate, 2012). Some evidence can be cited in support of this view of semantic CFDs as the apex of perceptive hierarchy. Coutanche and Thompson Schill (2014) presented an fMRI study that supported the hypothesis that semantics is based on the hierarchical integration of lower level (here: perceptive) features. They "found that the topdown retrieval of object knowledge leads to activation of shape-specific and color-specific codes in relevant specialized visual areas, as well as an object-identity code within left [anterior temporal lobe]" (p. 8, italics added). Collins and Olson (2014) proposed that visual face perception is hierarchically organized too, from lower-level face representations sensitive to viewpoint angle or head-orientation to viewpoint invariant face representations. They proposed that the ventral anterior temporal lobe is at the apex of the hierarchy and code for

4Incidentally, I'd like to notice that theoretical arguments are unfairly undervalued in the current practice of psychological science, and that a reappraisal of theory is essential.

Fernandez (2020) CFD model 
"view-invariant perceptual representations of faces", which are linked to "conceptual knowledge of individuals" (p.11).

In summary, it seems that the principle of iterative combination of CFDs might also account for semantic elements. One should stress that combination can involve many kinds of CFDs, from different sources, that is, from lower or from higher hierarchical levels, from perceptive or from executive axes. In particular, combined CFDs can also include lexical CFDs, whose role is probably important in semantic function.

\subsection{5 CFDs for high level perception and episodic memory}

A particularly interesting case is the one of episodic memory, as recent theoretical and empirical developments highlight its relations with the perceptive system. Put simply, episodic memory CFDs are claimed to be high-level perceptive CFDs. These CFDs would not be labeled episodic, strictly speaking. They would simply code for highly complex combinations of CFDs of various natures and perceptive modalities, at a very high hierarchical level in the perceptive part. As a result, an episodic memory can be considered as a high level percept, created during an event as the combination of a set of CFDs characterising the event, and reactivated by remembering. This idea that perception and memory emerge from mostly the same elements is a tenet of grounded cognition paradigm (e.g. Barsalou, 2008) and is compatible with the metatheoretical proposition of Fuster (1997) to shift the "focus from 'systems of memory' to the memory of systems" (p. 451; see also Gaffan, 2002). The approach defended here, is different however, and some aspects are mechanistically detailed. Let's consider theoretical and empirical data a bit more precisely.

First, how is it theoretically possible to construe episodic memories as high level perceptive elements? Simply, Ranganath (2010) argued that the medial temporal lobe participates in perception as well as in "episodic memory" through the binding of "objects and context". According to Diana, Yonelinas and Ranganath (2007), the contextual information would refer to "visual, spatial and semantic, gist or schema information that is peripheral to the study item" (p. 385). Similarly, it is proposed here that CFDs of medial temporal lobe neurons are simply more complex and heterogeneous than CFDs below in the hierarchy; they refer to events, characterized as combinations of spatially and temporally determined CFDs for objects (e.g., a wallet, a truck...), persons (e.g. Friedrich, Louise...), locations (e.g. at home, at work...), scenes (e.g. a bar, a factory...), etc. These CFDs might be all of the most complex CFDs in the brain, thanks to their position at the top of the perceptive part hierarchy -although some simpler CFDs might also be involved. A detailed account of the precise pattern of combination in various structures of the medial temporal lobe is out of the scope of the present article, but discussion can be found, for instance, in Ranganath (2010).

This theoretical proposition of is also supported by neurophysiological evidence. Indeed, neurophysiological and anatomical data support the idea that the most important episodic memory structure, medial temporal lobe (i.e., hippocampus proper, dentate gyrus, subicular complex, amygdala and perirhinal, entorhinal, and parahippocampal cortical fields; Squire et al., 2004) is also a good candidate for multimodal high level "perception", since many higher-order sensory cortices project onto the perirhinal cortex (Murray, Bussey, \& Saksida, 2007). Several authors argued that the "visual hierarchy" extends up to the hippocampus (Buckley, 2005; Buckley \& Gaffan, 2006; Bussey \& Saksida, 2007; Murray \& Bussey, 1999; Murray et al., 2007; Graham, Lee, \& Barense, 2008; Graham, Barense \& Lee, 2010; Riesenhuber \& Poggio, 1999; reviewed in Lee, Yeung \& Barense, 2012, or Baxter, 2009). Lee et al. (2012) suggested "that the hippocampus processes complex conjunctions of spatial features" (see also Cowell, Bussey \& Saksida, 2010; Saksida \& Bussey, 2010). Hippocampus (Paz, Gelbard-Sagiv, Mukamel, Michal Harel, Rafael Malach and Fried, 2010), and another medial temporal lobe structure, perirhinal cortex (Naya \& Suzuki, 2011), were 
also suggested to be implicated in coding sequences of sensory events. Hippocampus seems to be involved in encoding temporal ordering too (e.g. MacDonald, Lepage, Eden \& Eichenbaum, 2011; Manns, Howard \& Eichenbaum, 2007). This hypothesized multimodal convergence from different modalities in the medial temporal lobe was also supported by single-cell recordings in Humans achieved by Quian Quiroga, Kraskov, Koch, and Fried (2009). These authors further claimed that "the degree of multimodal invariance increases along the hierarchical structure within the [medial temporal lobe]" (p. 1308). Finally, GelbardSagiv Mukamel, Harel, Malach, and Fried (2008) recorded some neurons that were selectively active both during the viewing of a cinematic episode and during its free recall. This activation pattern suggests that these neurons actually might be the CFDs for the mnesic episode of the movie sequence. All these data considering medial temporal lobe as a perceptive structure are very stimulating and supportive of the present proposition, since the medial temporal lobe has also largely been considered a strictly mnesic structure (e.g. Squire et al., 2004).

\subsection{6 CFDs for words}

The language has a specific and major role in high level human cognition. Again, the elements of language, particularly words, are postulated to be hierarchically organized CFDs in various parts of the cognitive space, for instance, visual images of letters and words (Dehaene, Cohen, Sigman \& Vinckier, 2005), auditory images of phonemes or words (DeWitt \& Rauschecker, 2012). On the one hand, linguistic CFDs can be considered as perceptive (e.g. visual for letters or written words, auditive for phonemes or auditory words or segments, etc.) elements. On the other hand, they present the particularity to be associated with other CFDs, to wich they refer. For instance, CFDs for noun words are bidirectionally connected with the CFDs of the concepts or objects that they refer to. Concurrently, CFDs for word and / or phoneme motor articulation are predicted to lie in the executive system (Hickok, 2012). They are also hierarchically organized and are bidirectionally connected with their auditory and visual counterparts.

In the perceptive part of the neural network, the hierarchical combination principle appears able to account for many different (perceptive, conceptual, mnemonic..) categories, such as low and high-level perceptive categories, episodic and semantic memory categories...

Some categories of CFDs could not, however, be understood directly in this way. Cognitive functions related to working memory, executive functions and the use of goals and forwardthinking require to consider the "executive" part of the network, as suggested by Fuster (e.g. 2006).

2.1.2.4.2 Executive (motor)

The executive part of the system is claimed to be composed of CFDs associated with actions (in a large acceptation). The iterative hierarchical combination principle is claimed to link CFDs for simple (e.g. moving a digit upward) to complex (e.g. tinckling) movements, to actions (grasping a glass) to action plans and goals (e.g. going to the restaurant), procedures (cooking an egg or posing an operation) perhaps up to even more abstract goals (e.g. being and remaining attentive to everyone in a meeting). Following Fuster (2000, 2006), all the CFDs in this part are referred to as "executive", to highlight the assumed similarity between "concrete" motor CFDs and CFDs involved in more abstract actions. "Executive functions", or cognitive control, in its ordinary meaning, refers to control functions, and in psychological sciences to "the ability to coordinate thought and action and direct it toward obtaining goals" (Miller \& Wallis, 2009, p. 99). Beside this meaning, executive can also evoke the idea of acting, carrying out something. So it can refer to the axis that span from low-level motor CFDs to control and action CFDs. Here, control is supposed to be based most importantly on 
the activation of high level executive CFDs. The executive hierarchy, involving the frontal cortex, is based on the work of Fuster (2000, 2006), who claimed that the executive part is hierarchical, from "elementary muscular movements" at the bottom (primary motor cortex), to "more complex movements defined by goal and trajectory" (premotor cortex), to "broad schemas of action in skeletal and speech domains" (prefrontal cortex; Fuster, 2000, p. 66).

At the psychological level, cognitive control is addressed on the basis of Duncan and colleagues ideas about task model and goal neglect (Duncan et al., 1996, 2008). These authors suggested "that as new behavior is constructed, relevant facts, rules, and requirements must be organized into an effective control structure or mental program. We call this program a 'task model"' (Duncan et al., 2008, p. 133). In the present model, the goals and sub-goals of the task model are postulated to be implemented in executive CFDs. The activation of such a task model is supposed to be based on a large-scale resonant activation of relevant CFDs. Other kinds of CFDs are also probably involved for active maintenance, such as verbal CFDs (Cragg \& Nation, 2010), and emotional CFDs related to motivation (cf. O'Reilly et al., 2010).

Several models have suggested such a hierarchical organization of actions (e.g. Hamilton \& Grafton, 2007; Grafton \& Hamilton, 2007; Cooper \& Shallice, 2000, 2006). Here, it is assumed that most actions (from the simplest and most concrete to the most abstract ones) can be composed through the combination of a spatiotemporally organized combination of lower level CFDs (exactly as for perceptive CFDs). For instance, the potential executive goal "make a sandwich" would be at a higher hierarchical location (in the cognitive space) than the goal "slice bread". The reason why is not, strictly speaking, related to a conceptual principle like "abstraction", but is grounded into the mechanisms of the iterative hierarchical combination. Again, this principle is submitted to activation contingencies of CFDs that combine themselves. This might participate to explain why the organization of the motor cortex does not follow a strict abstract hierarchy of complexity, but is rather dependent on the idiosyncratic (or species-specific) behavioral repertoire of the animal (Graziano, 2006, 2016).

This cognitive view is supported by many neuroimaging data. Fuster (2000, 2006) claimed that frontal lobe structures subtended what he called "executive memory", that is, memory for actions. Conceptually, a concrete to abstract executive hierarchy is supported by fMRI studies (e.g. Koechlin \& Jubault, 2006, Fuster, 2004). Many authors provided evidence for a hierarchical organization of action goal representations in the rostro-caudal axis of the frontal lobe (Badre, 2008; Badre \& D'Esposito, 2009; Botvinick, 2008; Christoff, Keramatian, Gordon, Smith, Mädler, 2009; Koechlin, Ody, Kouneiher, 2003; Koechlin \& Summerfield, 2007; O'Reilly, 2010), although the very nature of this "hierarchy" is debated (e.g. Uithol, van Rooij, Bekkering, \& Haselagger, 2012). More and more complex / abstract actions are coded as one moves back from the primary motor cortex toward the premotor cortex, and then the frontal pole.

At the level of single units too, some studies showed that lateral prefontal cortex neurons might respond to abstract goals, such as the movement of a cursor independent on the biological movement to be performed (Saito, Mushiake, Sakamoto, Itoyama, Tanj, 2005) or a specific category of behavioral sequences, independent of the specific embodiment of these sequences (Shima, Isoda, Mushiake, \& Tanji, 2007; cf. Tanji, Shima, \& Mushiake, 2007, for review). Wallis, Anderson and Miller (2001) recorded single neurons coding for abstract rules in the prefrontal cortex of rhesus monkeys. The case of mirror neurons is also relevant for the present issue (Rizzolatti \& Craighero, 2004; Hickok, 2015). Some of these well-known mirror neurons appeared to code for particular categories of actions, or for some action goals, independently of the subject of the action (i.e. the animal itself or a perceived fellow congeneric). This represents an impressive example of abstraction in the domain of action. This is coherent with the present view about executive CFDs, and with the hypothesis that the same CFDs should be activated in various cognitive processes, for instance imaging, 
remembering, or actually achieving a same given action (cf. the notion of memory of systems, Fuster, 1997).

Along the rostro-caudal / abstract-concrete axis, an other organization principle has been proposed. O'Reilly (2010, see also, Sala et al., 2003) proposed a What / How distinction along the ventro-dorsal axis of the PFC, which is reminiscent of the What / How pathway dichotomy in the perceptive system (Goodale \& Milner, 1992; Goodale, 2011). Such similarity between the executive and perceptive parts would perfectly fit the present framework, particularly in reason of the perception-action cycle postulate. Indeed, following this postulate, organization schemes in perceptive and executive parts are supposed to influence each other quite strongly, and so to present important similarities.

In brief, in the executive part like in the perceptive part, more abstract / complex CFDs are claimed to emerge through the weighted spatiotemporal combination of other (mostly lower level) CFDs, and this point is supported theoretically and empirically. According to the perception - action cycle principle (expressed by the location of these executive CFDs in the cognitive space), executive CFDs also receive afferent connections from the "other part" (i.e., the perceptive part).

\subsection{Links between perceptive and executive CFDs}

Perceptive and executive parts are supposed to be strongly connected, even if they can be theoretically distinguished. Visually-driven eye movement control can illustrate and highlight this issue of interactions between perceptive and executive CFDs. In the perceptive part, some maps code for space in a retinotopic way (Silver \& Kastner, 2009). These spatial maps are supposed to emerge as (lower-level) visual CFDs combine together, independently of the characteristics they code for, and in a spatially selective manner. Since these combining CFDs are mostly visual, the reference space can remain retinotopic. On the other hand, in the executive part, some CFDs are organized in similar maps, but coding for eye movements (e.g. in frontal eye field or superior colliculus, Pierrot-Deseilligny et al., 2004). These maps are coded in a mototopic way (Stein \& Stanford, 2013), which is constrained by the approximately two-dimensional- physical possibilities for the eye to be moved. Both maps (visuospatial and spatio-motor) are directly connected, anatomically and physiologically (Pierrot-Deseilligny et al., 2004) and are concurrently involved in similar functions, most notably visual attention (Shipp, 2004), and eye-movement control (Pierrot-Deseilligny et al., 2004). It is simply hypothesized that CFDs of each of these maps are connected in a spatiallyspecific way (perhaps also through subcortical "visuospatial" and / or motoric structures, such as SC or pulvinar, too; cf. Shipp, 2003). These perceptivo-executive connections, related to the perception-action cycle, are considered at the core of several cognitive processes and behavioral phenomena, such as visuospatial selective attention and eye-movement control (endogenous and exogenous; Iacoboni, 2000), or visual stability (Cavanagh, Hunt, Afraz, \& Rolfs, 2010). Such structural connections and corollary functional interactions (activation diffusion) are likely to be qualitatively and quantitatively important in cognitive functioning, as suggested, among other things, by the perception-action cycle principle.

In addition, the dense connections between perceptive and executive CFDs is also highly relevant to the issue of cognitive control. All CFDs constituting the goals and subgoals of the task are connected with executive CFDs and probably most of them are connected with perceptive CFDs, at various hierarchical levels. Indeed, some actions are associated with quite specific and "concrete" percepts (e.g. 'basketball playing' with a 'basketball'; 'smoking' with various kinds of 'pipes' or 'cigarettes'), or mostly with more abstract concepts (e.g. 'thinking' with 'concepts', 'ideas'...). These associations are implemented in more or less strong connections. This architectural point might form the principled and mechanistic basis of often hypothesized "top-down" signals for cognitive control (e.g. Miller \& Cohen, 2001). Finally, 
some connections might also be constructed on line, in more plastic regions, to represent new rules demanded by the task at hand (Wallis et al., 2001).

\subsection{Dynamics of cognition}

\subsubsection{General considerations: about a structured dynamic system}

\subsubsection{Cognition emerges from dynamic activation...}

Having described the general architecture (or structure) of the system, one could finally turn to its dynamics of activation, which is directly related to and dependent on the underlying architecture. First, cognition is considered as a continuous process, rather than a sequence of discrete representations. This is also a central tenet of the dynamical systems theory. In this research field, the dynamics of cognition is emphasized, at the expense of the structural architecture of cognitive systems. This emphasis is both permitted and constrained by the mathematical tools at the basis of this approach (Beer, 2000). Mental states are described as points in a high-dimensional geometrical state space (Spivey \& Dale, 2006). In this space, distance is related to similarity between mental states, considered globally. These points can only move continuously from one location to an other. Some locations in the state space can exert an attraction on the (mental-state) points, which attraction falls gradually as distance from the attractor increases. "These regions are called attractor basins, and they correspond to patterns of neuronal activity that are elicited when someone is [e.g.] recognizing a spoken word or a visual object" (Spivey \& Dale, 2006, p. 207). Cognition can be considered as "a continuously changing pattern of neuronal activity" (Spivey \& Dale, 2006, p. 207). The dynamical system approach gets credit for stressing the dynamical aspect of cognition and the view of cognition as a continuous process.

\subsubsection{2 $\quad$...in a structured network of cognitive features}

One important criticism can be made against the dynamical system approach, however. Possibly due to its mathematical formalism and to its emphasis on time, it overlooked the fundamental role of the architecture, the structure, of the mind-brain. Indeed, in dynamical systems, each state of activation of the network is represented by a single point. Representing the whole cognitive system state as a point (in a cognitive state space) prevents from directly addressing the spatial architecture of the cognitive system, and its foremost importance, stressed above. Actually, at least some dynamical system theories do obviously assume real neural networks to underlie the activation states. Most often, however, the network architecture is simply not considered in itself. The problem is that it is probably impossible to put the whole "cognitive state" of such a complex natural nonlinear system into a sufficiently simple mathematical variable (Eliasmith, 1996). Therefore, although intellectually stimulating and heuristic, dynamical system theories are considered insufficient to fully address the mind-brain problem, and a model concerned with the brain spatial structure should be prefered. Indeed, the spatial dimension is essential for understanding cognition. First, I argue that understanding cognitive processes must be based on the isomorphism between cognitive and neural spaces. Second, this is precisely the CFD location in the cognitive space that is thought to underpin its cognitive value. Third, and most importantly, this is the spatial pattern of connectivity of activated CFDs that is assumed to explain why cognitive activity goes the way it goes, since cognition is thought to be reducible to the activation spread in the cognitive space. Thus, it is necessary, I believe, to consider -at every moment- the spatial distribution of CFDs' activation, as well as its dynamical evolution over time, in order to genuinely unravel the mechanics of cognition. 


\subsubsection{Dynamics can be derived from the pattern of connectivity}

The activations (firing of neurons' large assemblies) spread in the network according to the architecture of this latter, that is, according to the pattern of connections and the "conductance" of these connections. According to the characteristics of the current activations, to the connection pattern, to the strengths of the connections, the activation will diffuse more or less intensely from one part of the cognitive space to some or others of the connected ones.

For instance, consider that a known stimulus is visually presented. Perceptive regions first begin to be activated, then rapidly spread activation along visual pathways (e.g. ventral and dorsal), because the activations are strong and temporally congruent enough and because these connections are highly effective (due to past perceptive learning, in early development or after). Therefore, the occipito-temporal lobe is rapidly activated (as reviewed in Bullier, 2001). To rephrase it at a more fine-grained level: this propagation is determined by both the pattern of activation at each hierarchical level and the pattern of connections. For perception of a well-known stimulus, propagation would be fluent because, at each level $\mathrm{N}$, the set of activated CFDs would be very similar to the optimal set of CFDs composing some level $\mathrm{N}+1$ CFDs. Level N+1 CFDs would thus easily be activated, and activation would easily propagate to this level, and so on for successive levels.

Within a given part (i.e. perceptive or executive), the dynamics of activation can thus grossly be derived from the connectivity of CFDs. This is relatively straightforward for nonambiguous actions and stimuli, at least up to mid-range hierarchical levels. For more complex computations (e.g. ambiguous stimuli, complex action...), spread of activation is supposed to be more "difficult" and slower. More feedback-feedforward interactions and resonance (Grossberg, 2013; cf. Section 2.2.2.1.7 Bidirectionality and adaptive resonance) are needed to allow activation spread continuation. Indeed, ambiguous or noisy stimulus would not activate a set of low-level CFDs optimal for directly and selectively activate the correct target (e.g. mid-level) CFD. Some principles of activation dynamics, thought to emerge from the connectivity patterns of the brain, are presented below.

\subsubsection{Principles of activation diffusion in the neural network}

\subsubsection{Basic rules of transmission}

As a proxy, the general law of the cognitive space network stipulates that a neuron firing rate will depend on the spatiotemporal weighted sum of all the incoming signals (Feldman \& Ballard, 1982). This is a simple rule of connectionist networks, given by the activation function of each neuron. Activation spreads in the network as long as the nodes of the network (the neurons or CFDs, according to the viewpoint) receive a total amount of activity that exceeds their respective current thresholds. Two illustrative examples of this general principle deserve to be described, since they have particular interest at the cognitive level, namely "bottom-up" and "top-down" activation spread (Bullier, 2001).

\subsection{Feedforward diffusion \& "exogenous processes"}

First, propagation can move bottom-up, that is, from neurons at a low hierarchical level to neurons at a higher hierarchical level. Typically (e.g. during perception), activation can "begin" at the level of sensors and propagate towards higher and higher levels through

5Actually, activation never "begins", since the network as a whole is always active in some way (e.g. Buckner, Andrews-Hanna, \& Schacter, 2008). The term is used for simplicity to describe the first activation more specifically associated with the processes we are 
successive convergent connections. A given set of CFDs can activate a higher level CFD on which they project. This is a bottom-up process, which might occur, to give an imaginary example, when three CFDs coding for three contiguous "oriented bars" forming a triangle would project on a hypothetical "triangle" CFD.

Such feed-forward sweep of activation through the network can in some cases activate very quickly some high-level categorical characteristics (e.g. Thorpe et al., 1996). This socalled "feedforward" sweep, however, is not necessarily restricted to the perceptive part. The activation would spread as far as the activation state and the connectivity pattern would conjointly allow. For instance, if a sufficient set of CFDs is activated and if the connections of the activated set of CFDs are efficient toward some executive CFDs, one might predict a rapid perceptually-based (or stimulus-driven) action to occur. This might be the case of very rapid simple categorization saccades (Kirchner \& Thorpe, 2006; Crouzet, Kirchner, \& Thorpe, 2006), or even more likely of exogenous captures of the eyes by salient new objects (Theeuwes, Kramer, Hahn, \& Irvin, 1998), for instance. For such situations, there might exist a rather direct, sufficiently "conductant" pathway that allows to connect sensors to effectors. Such very efficient perceptive-to-executive spreads of activation are certainly not always possible. Some stimuli or scenes are not informative enough, and some categories are too much "high-level", perhaps too much abstract (e.g. honest) to be categorized efficiently so fast. "Feedback" influences are usually also necessary, and their influence is probably pervasive (e.g. Bar, 2004; Deco \& Rolls, 2005; Hedgé \& Felleman, 2007; Lamme \& Roelfsema, 2000).

\subsection{Feedback diffusion \& "endogenous cognitive processes"}

\subsection{Perceptive top-down influences}

So top-down influences are also considered. They correspond to descending activations (from higher to lower hierarchical levels). The most famous top-down influences, but clearly not the only ones, are probably those from working memory, addressed in the next section (2.2.2.1.2.2 Working memory influences, cf. Miller \& Cohen, 2001). Top-down influences are also, for instance, involved in most "perceptive processes" (not involving working memory). Indeed, when a CFD is activated by ascending projections, it will likely send ascending projections in its turn. This is the core of the bottom-up perceptive mechanisms, progressively extracting information and analyzing the percept (Riesenhuber \& Poggio, 2000). However, the CFD at hand projects towards every CFD to which it is directionally connected. It therefore projects not only towards higher levels, but also toward lower levels CFDs. Particularly, the CFDs recurrently project toward the CFDs that precisely projected toward them (backward projections), according to the general principle of bidirectional connectivity (Rolls, 2008). Therefore, an activated CFD will enhance, backward, the activation of the CFDs of which it is "composed" -i.e. which projected on it (see Figure 3). This is a kind of top-down mechanism, which would facilitate the perception, by backward reinforcing activation of CFDs corresponding most closely to known perceptual elements (coded by existing superordinate CFDs). The generalized recurrent activation is useful for ambiguous or blurred, not well known, etc., stimuli, in that it facilitates the selection of one CFD among several possible candidates, through a mechanism akin to what Grossberg (2013) called "resonance".

interested in. But it is probably safe to always keep in mind that the brain is probably never in a neutral, inactive state. 
1.

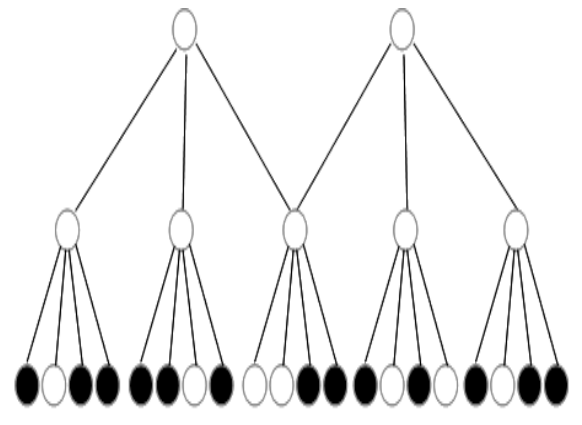

2.

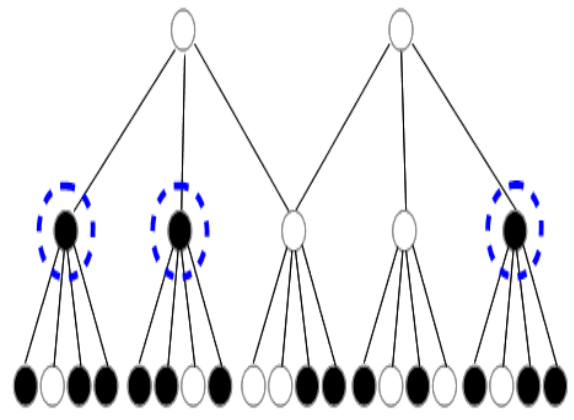

3.

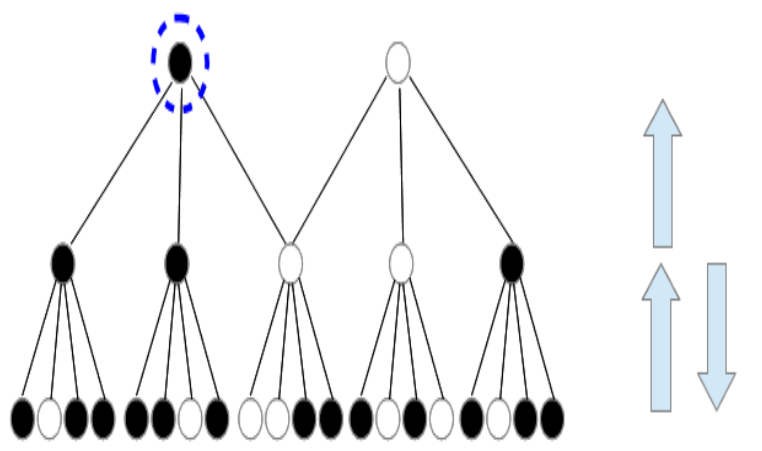

4.

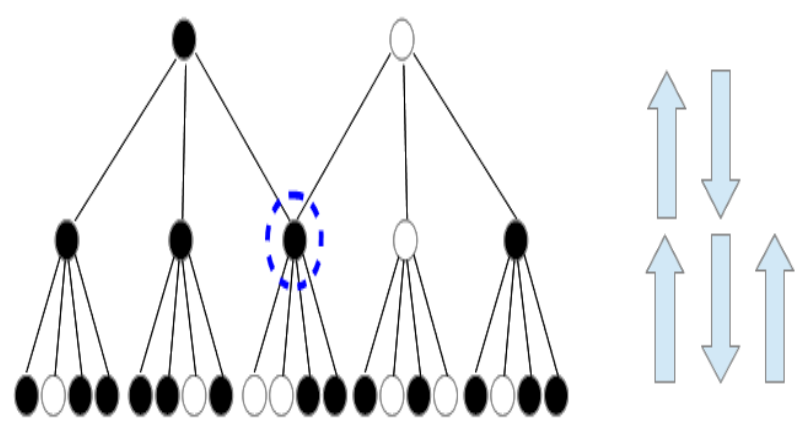

5.
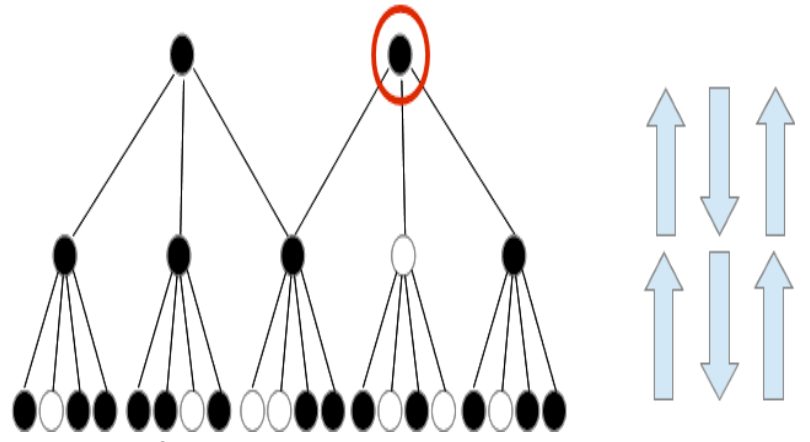

Illustration 4: This figure illustrates a predominantly bottomup activation spread, with consecutive recurrent activation spread. While the first activation sweep toward the third hierarchical level (panel 3) was insufficient to activate the top right CFD, recurrent activation spread (panel 4) allowed to activate it (panel 5). Information stored in the CFDs' patterns of connections allowed to efficiently analyze the perceived scene. 
The activation is thought to propagate through cascades between CFDs of various levels, in both directions. The importance of top-down signals is underscored by several authors, with various conceptualizations (e.g. Ahissar \& Hochstein, 2004; Bar, 2003; 2004; Deco \& Rolls, 2004; Hamker, 2005; Kveraga, Ghuman \& Bar, 2007; Lamme \& Roelfsema, 2000). Here, top-down and bottom-up signals can still be theoretically differentiated but they are inextricably entangled: it is thus probably impossible to associate concrete empirical phenomena with one or the other activation spread direction, except in some extreme situations (e.g., perhaps, Thorpe et al., 1996).

This top-down process is not qualitatively different from the working memory descending activation, but can have a different spatiotemporal pattern of influence. While working memory is mostly a sustained, long-range, descending activation that often begins prior to the stimulation (e.g. Serences \& Boynton, 2007), the perceptive top-down process is usually a more short-range signal (though not necessarily, cf. e.g. Kveraga, Boshyan \& Bar, 2007), which begins after the stimulation and is based on reciprocal connections.

2.2.2.1.2. Working memory influences

Maintained activations related with working-memory can also send descending excitatory projections toward lower level perceptive and/or executive CFDs. For instance, if "triangle" is a relevant CFD for the subject at the time, for instance as a target in an upcoming visual search task, it might therefore be maintained active thanks to working memory processes. Consequently, since some "oriented bars" CFDs receive descending activity (from this perceptive "triangle" CFD, and from farther apart), they would be more easily or more quickly activated by ascending projections. Indeed, a lower total amount of ascending activations might be sufficient, thanks to the added descending signals.

In the present model, working memory, or executive control, is based on two constructs: task model and template. Task model is composed by the CFDs for goals, subgoal, rules, etc., related with the task at hand (Duncan et al., 2008). Thus, the task model is thought to subtend the executive functions of the working memory. The template refers to the material, or the "representation" that has to be worked on, and is composed of perceptive and executive CFDs (cf. Duncan \& Humphreys, 1989). The template represents the mnesic content of the working memory. Task model and template are distinct assemblies of CFDs but are not qualitatively different (since both are made of CFDs). It is claimed: (a) that the task model and the template(s) are necessarily connected ${ }^{6}$; (b) that the resonant state of the task model (possibly with the help of lexical and "motivational-emotional" CFDs) permits the maintained activation of the template; and (c) that this pre-activation of the template facilitates efficient activation spreads during the related upcoming cognitive processes.

The CFDs for the Task model and template might just differ in location in the system. In classical cognitive theories (e.g. Baddeley, 2012), the situation is regarded in a very different -i.e. dichotomous- way, with a content on the one hand and a (executive) system dedicated to "manipulating" this content on the other hand. This difference illustrates one particularity of the present model.

Verbal working memory is probably a very important function in Humans, as it might be engaged in many cognitive actions. Following Acheson and colleagues' insights (Acheson \& McDonald, 2009; Acheson, Hamidi, Binder \& Postle, 2011), it is proposed that verbal working memory might emerge from a large scale resonant state engaging CFDs for auditory and motor word forms, which are hypothesized to be strongly connected, at least indirectly.

6Indeed, it was argued above (2.1.2.2.4.3 Links between perceptive and executive CFDs), that executive CFDs are expected to be connected with associated perceptive CFDs.

Fernandez (2020) CFD model 
In considering how goals, templates, and so on, are maintained in working memory, one should also consider that the environment can also drive important activation patterns, in a way that is often relevant for the task at hand. For instance, seeing the display of a visual search task would probably activate CFDs recently activated and thus the associated task goals' CFDs, therefore triggering or facilitating the reactivation of the task set resonant state. This emphasis put on the external world for cognition is reminiscent of situated cognition issues (cf. e.g. Robbins \& Aydede, 2009; O'Reagan \& Noë, 2001).

\subsection{Short term facilitation}

Coherently with neuroscientific data (e.g. Zucker \& Regehr, 2002), it is hypothesized that an activated neuron will be more prone to be re-activated (i.e. decreased threshold) for a short period after. This point can help accounting for various kinds of (spatial, featural...) priming. Some authors also proposed that a synaptic facilitation is the basis of short term memory (e.g. Mongillo, Barak \& Tsodyks, 2008). The model is agnostic regarding the precise mechanisms at the neurophysiological level of analysis. Both mechanisms might allow the same kind of activation dynamics change, or each mechanism might support a distinct kind of short term memory process.

\subsection{The perception-action principle}

The perceptive / executive dichotomy is also important relative to the diffusion of activation. Indeed, the main trend of activation spread roughly follows a perception-action cycle. This perception-action cycle principle was proposed by Von Uexküll (1926) and developed by Fuster (2004, 2006), who stated that:

"The behavior of an organism is subject to a continuous circular flow of information between itself and its environment. Environmental stimuli are received and processed by sensory structures; as a result of sensory processing, actions are generated that cause certain changes in the environment, which lead to new sensory input, and so on" (Fuster, 2006, p. 130).

This does not mean that there is a linear and unequivocal direction of activation spread. Rather, this direction of activation represents the average direction, the trend, and it can be metaphorically compared to a slowly moving bee swarm: bees are moving in many directions but, as a whole, the swarm has a form and has a direction of movement; the swarm can also split in several ones, or vice-versa several swarms can conglomerate.

This idea of a perception-action cycle is not a metaphysical one, but is grounded on concrete mechanisms. It is simply given by mechanistic characteristics of the most fundamental elements of the system (in term of simplicity and ontogeny): the sensors and effectors. Indeed, sensors are very predominantly activated from the outside, effectors from the inside. This is obviously related to the nature of perception and action. From this very simple initial facts, the resulting activation dynamics, and related statistical activation contingencies are assumed to determine the development of a pattern of connectivity in which the weights are generally stronger in one direction than in other. This should explain why, in adults systems, the global connection characteristics make the activation spread more efficient in the perception-to-action direction.

\subsection{Horizontal inhibitory connections}

In addition to ascending and descending excitatory connections, horizontal inhibitory connections are also postulated between incompatible CFDs. Such inhibition is postulated to occur only (or mostly) at a given hierarchical level, not (or rarely) between levels (cf. Aron, 2007, for possible exceptions). It is supposed to subtend "competition" between some CFDs. 
Desimone and Duncan (1995, Duncan et al., 1997; Duncan, 2006) have developed and defended a "biased competition" model of attention. Duncan (2006) claimed (1) that "stimuli compete for representation" in the brain (p. 6); (2) that this competition is integrated across different parts of the brain network ("As one object's representation gains dominance in any one part of the network, so its representation is strengthened elsewhere", p. 9); and (3) that this competition can be biased top-down. These three principles (competition, inter-system integration, top-down biasing) inspired the present model, although they were remodeled in the CFD framework.

In the present model, competition is thought to occur only (or very predominantly) within cognitive maps (cf. Franconeri et al., 2014), at every location in the cognitive space, and to decrease as a function of distance between the competing CFDs ${ }^{7}$. First, between-map inhibition would necessitate a very dense wiring, and would thus be neurophysiologically prohibitively expansive. Yet such wiring seems not necessary, from a functional viewpoint. Indeed, inhibition between maps can be functionally replaced by between-map excitatory connections for associated CFDs plus within-map inhibition between incompatible CFDs (Figure 4). Such a pattern of connection involves a much less dense wiring. That's why between-map inhibition is not postulated here, though it might possibly exist in some specific cases (Aron, 2007).

Second, competition is not restricted to the perceptive system, but is supposed to occur in any cognitive map, and to concern action too (Cisek, 2007; Cisek \& Kalaska, 2010) and cognitive functions involving high level CFDs, such as semantic memory, executive task goal CFDs (Courtney, Roth \& Sala, 2007), and so on. The reason why is simply that every cognitive map in the system is supposed to be similar in nature, and organized according to essentially identical principles.

Third, competition is claimed to decrease with distance (as in Trappenberg et al., 2001; Wang, Kruijne, \& Theeuwes, 2012) because (a) CFDs are spatially organized by similarity within a map and (b) similar CFDs are more prone to be competitive. The similarity-intopography principle (Simmons \& Barsalou, 2003; cf. section 2.3.3.2 Map construction principles) is generalized to the whole cognitive system (not limited to specific convergence zones, as in Simmons \& Barsalou, 2003). According to this (extended) similarity-intopography principle, CFDs are spatially organized in cognitive maps as a function of their similarity.

The precise neurophysiological principles accounting for competition are not fully known, and out of the scope of this article. As a putative mechanism, it can be postulated that CFDs compete in the networks trough inhibitory lateral connections.

To our knowledge, there is no direct and unequivocal evidence that the competition in the brain is subtended by such inhibitory mechanisms. There only seems to be a competition based on lateral inhibitory connections (e.g. Knierim \& Van Essen, 1992, Beck \& Kastner, 2005). The special kind of connectivity implementing competition, however, is not a strong claim of the model.

In summary, a general principle of competition is postulated. It is thought to occur for pairs of CFDs whose concurrent activation is impeded or opposed to by activations from the rest of the system. The notions of similarity and compatibility are only helpful to apprehend the model's functioning, they are not explanatory. The explanation, in last instance, lies in the concrete mechanisms of activity-dependent synaptic modification (cf. section 2.2.3.2 Map construction principles).

7Remember that distance is inversely related to similarity, cf. 2.2.3.2 Map construction principles.

Fernandez (2020) CFD model 


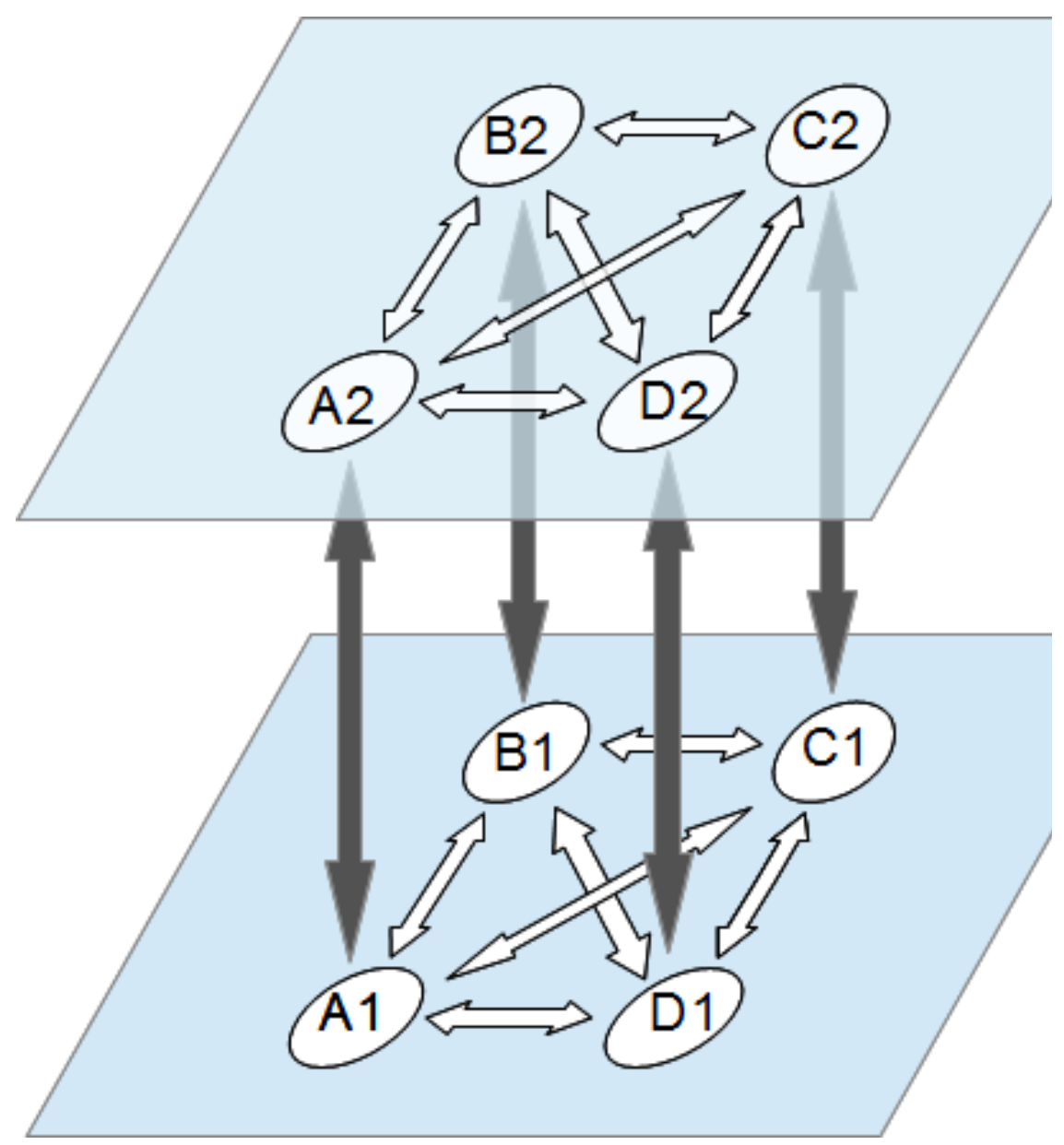

Illustration 5: This figure represents a general pattern of connections between CFDs of two maps of successive levels $(N$ and $N+1)$. Within a given map, connections are predominantly inhibitory (white arrows), allowing competition. Between maps of distinct levels, CFDs are predominantly connected by excitatory bidirectional connections (black arrows). This pattern of connection allows associated CFDs (e.g. A1 and A2, B1 and B2) to share their competitive advantages or disadvantages (as formulated by Duncan, 2006).

\subsection{Horizontal excitatory connections}

Horizontal connections are probably not all inhibitory (Lamme \& Roelfsama, 2000). Indeed, similar CFDs can be active simultaneously for different spatial locations, given the spatial selectivity that pervades the whole visual system (although with decreasing importance as one move upwards the ventral stream, Rolls, 2008). Neurons coding for similar CFDs can thus have excitatory connections too (Lamme \& Roelfsama, 2000). No strong claim is made about that, neither. Some features of a same hierarchical level might have positive statistical contingency, and have developed excitatory connections. As elsewhere in the article, the principle proposed here is expected to rely on simpler concrete mechanisms.

\subsection{Bidirectionality and adaptive resonance}

The basic rules of transmission were just succinctly described. However, the dynamics of the network is more than the sum of some simple activation spread principles (feedforward, feedback, lateral inhibition...). Some more complex patterns of activation would mechanically emerge from the dynamical activation of the network on the basis of these simple rules. One 
important concept in this regard is resonance (Grossberg, 2013). Resonance refers to the activation pattern of mutually excitatory neurons, or of an assembly of neurons, that allows them to remain active as a whole for some time. In a simplified paradigmatic case, consider two neurons projecting excitatory connections one towards another. If each projection suffices to activate the other neuron, then both neurons might engage in a maintained dialogue: neuron A triggers neuron $\mathrm{B}$, which triggers neuron $\mathrm{A}$, and so on ${ }^{8}$.

Such an over-simplistic structure is implausible as such. However, on the basis of this very mechanism, larger assemblies of CFDs, possibly over several levels (and involving both perceptive and executive CFDs), can more probably enter a resonant state. Indeed, one CFD alone is unlikely to activate another CFD, yet several CFDs are much more likely to do so. Consider a group of CFDs (at level $n$ ) activating a second group of CFDs (at level $n+1$ ). These latter CFDs (at level $n+1$ ) might in turn suffice to (re-)activate the first group of CFDs (at level $n$ ), or at least a part of them. Therefore, resonance is more likely to occur through larger sets of CFDs. The resonance phenomenon is rendered possible by neural connections being usually bidirectional in the brain (Felleman \& Van Essen, 1991; Lamme, Super \& Spekreijse, 1998; O'Reilly, 1998; Rolls, 2008). That is, when a CFD A projects onto a CFD B, this latter (CFD $B$ ) usually sends a reciprocal connection toward the former (CFD $A$ ), although it usually ends at a different cortical layer (Grossberg \& Pearson, 2008). This principle of resonance is supposed to be involved in many different cognitive processes, such as perception (Pollen, 1999), or short term memory maintaining (Grossberg, 2013). Given the hierarchical organization of the network, numerous CFDs at several stages might engage in a large resonant state.

\subsection{Multiple loci of activation}

Now is addressed one crucial reason why spatial architecture of the network is important to consider, in addition to the temporal dynamics. In the network, several (distinct) sets of CFDs can engage in simultaneous resonant states. A resonant state can remain active at one zone while activation spreads toward a distinct zone of the neural / cognitive space. The merging, of both assemblies (or "clouds") of CFDs, can facilitate, or allow activation spread according to a path which would otherwise not be activated (e.g. because afferent signals are insufficient at this point). This idea of distinct loci of activation in the cognitive space can help understanding cognitive processing, for instance working memory and its influence on perception or attention. This case study would deserve to be developed more thoroughly and is schematically illustrated in Figure 5.

\subsubsection{Development}

\subsubsection{Development of the neural network / cognitive space}

The activation dynamics addressed above referred to how the states of activation varied through short time scales, from milliseconds to minutes (cf. Thelen, 1995). Larger time scales were not yet addressed. Considering longer time scales, from minutes to years, would however be very informative, in particular, but not only, for the first stages of the brain development (Karmiloff-Smith, 1998). Indeed, on might argue that the general architecture of the mind-brain can only be understood in detail in the light of its ontogenesis. Some fragrances of constructivism (e.g. neo-constructivism, Newcombe, 2011; neuroconstructivism, Sirois et al., 2008) would therefore be a useful approach to address the architecture of the

8For simplicity, the possibility that threshold increases with continuous activation is not considered here. 


\section{Executive part Perceptive part}
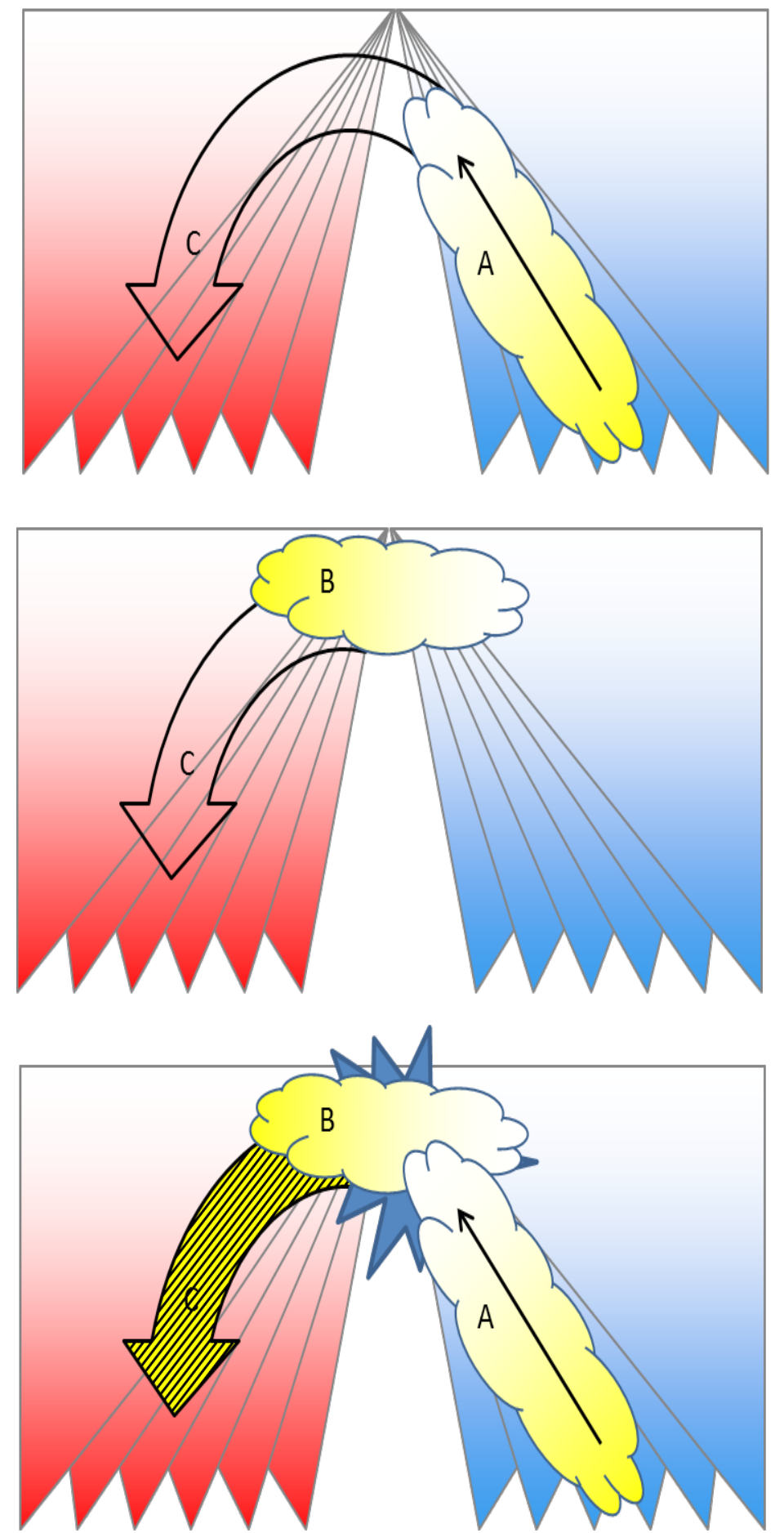

Illustration 6: This figure illustrates schematically how task-related CFDs (e.g. task goals or target templates) might mechanistically lead to a response action. Predominantly bottom-up activation spread lead to activation of executive CFDs that already receive top-down activations from an internal resonant CFDs assembly (bottom panel). Only the combination of both groups of afferences allows to exceed these CFDs thresholds and allows activation spread toward the executive part of the cognitive space (bottom panel, compared upper panels). Here, only the general principle is represented, very schematically. 
cognitive space. Indeed, some development of a system is out of range as long as the necessary structures are not sufficiently developed and need further construction of intermediate structural components, both at cognitive and neural levels (cf. Sirois et al., 2008).

The constructivist principles are straightforwardly applied in the development of the hierarchical organization of the neural network. The perceptive axis can be addressed as a tentative example. CFDs are created and tuned progressively, through a roughly hierarchical temporal progression. The reason why is the following. The construction, or tuning, of CFDs is based on statistical contingencies of other CFDs' activations. Let's consider CFDs of a given hierarchical level of complexity. Statistical contingencies of co-activations depend on the relative complexity of the target CFD. If this target CFD is just a bit more complex, statistical co-activation contingencies of composing CFDs are likely to be quite high. However, co-activations contingencies would decrease drastically as the difference in complexity between source CFDs and target CFD increases.

Let's take a concrete example derived from the Hubel and Wiesel model. "Sensory cells" (e.g. ON/OFF cells), through the statistical regularities of their activation, might give rise to CFDs for a just higher hierarchical level (e.g. a simple cell for oriented bar). On the contrary, it is probably impossible to find a set of those "sensory cells" whose activation is statistically regular enough to be strongly correlated with, for instance,te percept of a whole "dog". The development of intermediate levels is necessary for more abstract-complex CFDs to be constructed through combinations based on statistical regularities (of CFDs activations). A CFD for 'dog' would indeed be more easily expected through the combination of more complex and abstract mid-level CFDs (such as, hypothetically: (four) legs, hairs, barking sound, etc.), because these CFDs appear together with a much more higher and consistent statistical regularity. Thus, the model structurally predicts that higher level CFDs will emerge later than lower level ones -i.e. only when lower level CFDs have been constructed, or tuned.

The example presented above is probably over-simplistic and unrealistic. It should not mask, among other things, the importance of action in the construction of the cognitive system, as defended in constructivist theories (e.g. Newcombe, 2011). Strong connection patterns between perceptive and executive CFDs are essential to the structuration of the neural-cognitive system, particularly for cognitive control. Executive afferences are also postulated to participate in perceptive CFDs construction, and vice-versa. Indeed, following to the perception-action cycle (Fuster, 2006; Uexküll, 1926), perceptive and executive CFDs are densely connected. This issue will likely have to be developed. Finally, the possibility that new CFDs are created on the basis of adult neurogenesis and integrated in some locations, should also be considered.

The present model can also be said neuroconstructivist, as it considers "psychological development as entwined with the mechanistical and morphological aspects of brain development, from cell to brain to body" (Sirois et al., 2008, p. 322). Indeed, the notion of isomorphism between CFDs and their neuronal counterparts, defended here, perfectly fits this idea, and even offers a heuristic basis of it.

\subsubsection{Map construction principles}

The concept of map evoked above (section 2.1.2.2.3.1 Cognitive Maps) represents a useful approximation for the cognitive space architecture, but derives from more general principles. On the one hand, Simmons and Barsalou (2003) proposed the similarity-intopography principle, according to which "the proximity of two conjunctive neurons in a convergence zone increases with the similarity of the features they conjoin” (p. 451). On the other hand, Chklovskii and Koulakov (2004) proposed that "cortical maps may be viewed as solutions that minimize wiring cost for given intracortical connectivity” (p. 369). Adapted to 
the CFD model, this wiring cost minimization principle states that connection lengths between CFDs are minimized (modulo their weights). Both principles (wiring cost minimization and similarity-in-topography principles) are probably related, because if one solution minimizes the wiring cost for one CFD, then the solution for a similar CFD might often be similar, and therefore the two CFDs should be close. Indeed, "similar" CFDs are likely composed of similar CFDs. More precisely, if a CFD A is similar to a CFD B, then the set of CFDs composing the CFD A should be similar to the set of composing the CFD B: that is, a large proportion of the composing CFDs for CFD A are identical, or similar to CFDs for CFD B. And if the composing CFDs are similar, they are expected to be close from each other too (for similar reasons). The conceptual notion of similarity is directly related to concrete mechanisms, namely to the global similarity of the set of composing CFDs projecting on the target CFD.

At the beginning (and at the lowest level), the organization based on similarity is determined, or constrained by the spatial organization of sensors and effectors (indeed, two close somato-receptors, for instance, obviously code for close tactile stimuli). So the regression should continue down to sensor and effector CFDs, while the spatial organization of these latter is probably dependent on lower level processes (i.e. not cognitive: genetic, epigenetic, physiological). Thereafter, the "similarity" is defined, in mechanical terms, on the basis of statistical contingencies of co-activation. Two close receptive fields are more prone to be activated simultaneously than two distant receptive fields. Therefore, the CFDs associated with these two receptive fields will be closer in the cognitive (and neural) space, thanks to the wiring length minimization principle. Similarly, at higher levels, CFDs associated with similar sets of CFDs will be closer in the cognitive (and neural) space. The complex structure of the network is related to the complex patterns of CFDs' convergence, patterns of convergence which are themselves related with the statistical contingencies of these latter CFDs ${ }^{9}$.

\subsubsection{Development of competition}

Within a map, there are inhibitory connections between CFDs, achieving "competition" between CFDs, in the terms of Desimone and Duncan (1995). These inhibitions are claimed to be stronger between more similar incompatible CFDs (compatible similar CFDs should not inhibit each other, and thus are not addressed below). How does this pattern of inhibition emerges?

The hypothesized reason for this is that during perception a "competition" is more prone to occur between similar than dissimilar (incompatible) CFDs. Indeed, for a correctly activated CFD, a similar CFD is more prone to be incorrectly activated than a dissimilar one. From there, a Hebb-like activity-dependence rule of weight modification might lead to an increase of inhibitory connections. Consider two CFDs activated (one correctly and one incorrectly), each one sending inhibitory signals toward the other one. After a short time (involving descending signals and resonance), only the correctly activated CFD should remain active (as long as both CFDs refer to incompatible, or non-co-occurring events or stimuli). Therefore the course of their activation (i.e. concurrent activation rapidly followed by deactivation of one of the two CFDs) might reinforce the inhibitory connection from the "winner" towards the "looser", according to a Hebb-like activity-dependence rule. The reciprocal inhibitory connection is expected to be strengthened in situations where the roles are reversed. Physiologically, an inhibitory synapse might indeed be strengthened by a specific temporal pattern of firing through such rules (e.g. Spike Timing-Dependent Plasticity;

9Ultimately the statistical contingencies are related to the statistical characteristics of the world itself -more precisely the world as it is perceived.

Fernandez (2020) CFD model 
Caporale \& Dan, 2008), for instance an activation of both pre- and post-neurons, rapidly followed by a deactivation of the post-neurons (suggesting an efficient inhibition).

One functional reason of this competition for similar CFDs is avoiding simultaneous activation of incompatible CFDs and favoring the selection of the correct ones. Indeed, one location should not be perceived blue and red at the same time, for instance. Therefore, at one level of the hierarchy, blue and red CFDs for the same location should not be activated simultaneously. On the contrary, blue and red CFDs might be activated simultaneously for adjacent locations. Incompatible CFDs are mostly CFDs coding for real-world events that can theoretically not co-occur. But the same reasoning can work to some extent for abstract concepts too (e.g. war and peace).

To take an example in the domain of lecture, and assuming that written word CFDs exist (Dehaene, Cohen, Sigman, \& Vinckier, 2005), the CFD for the word 'cure' would be more similar, and more prone to be incorrectly activated simultaneously with the CFD for the word 'care' than to the CFD for the word 'salt' (considering only the most perceptive linguistic afferent at this point, not more abstract, semantic, afferents). Yet CFDs for 'cure' and for 'care' should obviously not both be activated if only the word 'care' is presented. Thus, their competitive inhibition should favor the activation of only one of them.

Similarity concerns both spatial and non-spatial dimensions, and can be of any nature, since it is only defined by the global similarity of the afferent CFDs' set. At the highest levels, incompatibility might even be defined on more abstract grounds; one can tentatively postulate semantic maps (cf. Ritter \& Kohonen, 1989).

\section{Conclusion}

The CFD model, developed in the present article, proposed as a basis of cognition a large set of CFDs, organized in a network. The complex architecture of this network (labeled cognitive space), with two main hierarchical parts, strongly connected with each other as parts of a Perception-Action cycle, is claimed to be at the basis of every cognitive act, since it determines how activation should spread among CFDs throughout the network.

The model provides some tools to address the mind-brain problem: cognitive and neural network are postulated to be isomorphic, with one CFD being represented in one (or at most a few) neurons. This perspective focuses on a more fine-grained level of analysis than the "cognitive function" level, which implies complex dynamic activation of large CFDs network, and is thus exceedingly difficult to apprehend directly. This might help putting order in the abundant neuroimaging data, perhaps more efficiently than the problematic usual tendency to localize cognitive functions (Fuster, 2003).

The model tries to describe the precise mechanisms (and the principles of these mechanisms) by which cognition emerges from the activation of the neural network. It is hoped that the integrative work attempted through this model, along with new notions or ideas, might help advancing in understanding cognitive mechanisms and how they are related to each other and to the brain. The present work aims at participating in tracing a heuristic research pathway for future cognitive neuroscience. Future works will address specific issues. 


\section{Bibliography}

Abrams, R., Klinger, M., \& Greenwald, A. (2002). Subliminal words activate semantic categories (not automated motor responses). Psychonomic Bulletin \& Review, 9(1), 100-106. doi: 10.3758/BF03196262

Acheson, D. J., Hamidi, M., Binder, J. R., \& Postle, B. R. (2011). A Common Neural Substrate for Language Production and Verbal Working Memory. Journal of Cognitive Neuroscience, 23(6), 1358-1367. doi:10.1162/jocn.2010.21519

Acheson, D. J., \& Macdonald, M. C. (2009). Twisting tongues and memories : Explorations of the relationship between language production and verbal working memory. Journal of Memory and Language, 60(3), 329-350. doi:10.1016/j.jml.2008.12.002

Ahissar, M., \& Hochstein, S. (2004). The reverse hierarchy theory of visual perceptual learning. Trends in Cognitive Sciences, 8(10), 457-64. doi:10.1016/j.tics.2004.08.011

Andersen, R. a, Snyder, L. H., Bradley, D. C., \& Xing, J. (1997). Multimodal representation of space in the posterior parietal cortex and its use in planning movements. Annual Review of Neuroscience, 20, 303-30. doi:10.1146/annurev.neuro.20.1.303

Anderson, B. (2011). There is no Such Thing as Attention. Frontiers in Psychology, 2(September), 246. doi:10.3389/fpsyg.2011.00246

Andrés, P. (2003). Frontal cortex as the central executive of working memory: time to revise our view. Cortex, 39(4), 871-895. doi:10.1016/S0010-9452(08)70868-2

Aron, A. R. (2007). The Neural Basis of Inhibition in Cognitive Control. The Neuroscientist, 13(3). doi:10.1177/1073858407299288

Baddeley, A. (2012). Working memory: Theories, models, and controversies. Annual review of psychology, 63, 1-29.

Baddeley, A. D., \& Hitch, G. J. (1974). Working memory. In G. H. Bowers (Ed.), The psychology of learning and motivation (pp. 47-90). New York: Academic Press.

Badre, D. (2008). Cognitive control, hierarchy, and the rostro-caudal organization of the frontal lobes. Trends in Cognitive Sciences, 12(5), 193-200. doi:10.1016/j.tics.2008.02.004

Badre, D., \& D'Esposito, M. (2009). Is the rostro-caudal axis of the frontal lobe hierarchical? Nature Reviews. Neuroscience, 10(9), 659-69. doi:10.1038/nrn2667

Bar, M. (2003). A cortical mechanism for triggering top-down facilitation in visual object recognition. Journal of cognitive neuroscience, 15(4), 600-609. 
Bar, M. (2004). Visual objects in context. Nature Reviews. Neuroscience, 5(8), 617-29. doi:10.1038/nrn1476

Barraclough, N. E., Xiao, D., Baker, C. I., Oram, M. W., \& Perrett, D. I. (2005). Integration of visual and auditory information by superior temporal sulcus neurons responsive to the sight of actions. Journal of Cognitive Neuroscience, 17(3), 377-391.

Barsalou, L. W. (2008). Grounded cognition. Annual Review of Psychology, 59, 617-45. doi:10.1146/annurev.psych.59.103006.093639

Barsalou, L.W. (2010). Grounded cognition: past, present, and future. Topics in Cognitive Science, 2(4), 716-724. doi:10.1111/j.1756-8765.2010.01115.x

Baxter, M. G. (2009). Involvement of medial temporal lobe structures in memory and perception. Neuron, 61(5), 667-77. doi:10.1016/j.neuron.2009.02.007

Beck, D. M., \& Kastner, S. (2005). Stimulus context modulates competition in human extrastriate cortex. Nature Neuroscience, 8(8), 1110-6. doi:10.1038/nn1501

Beer, R. (2000). Dynamical approaches to cognitive science. Trends in Cognitive Sciences, 4(3), 91-99. doi:10.1016/S1364-6613(99)01440-0

Botvinick, M. M. (2008). Hierarchical models of behavior and prefrontal function. Trends in Cognitive Sciences, 12(5), 201-8. doi:10.1016/j.tics.2008.02.009

Bowers, J. S. (2009). On the biological plausibility of grandmother cells: implications for neural network theories in psychology and neuroscience. Psychological Review, 116(1), 220-51. doi:10.1037/a0014462

Bowers, J. S. (2010). More on grandmother cells and the biological implausibility of PDP models of cognition: a reply to Plaut and McClelland (2010) and Quian Quiroga and Kreiman (2010). Psychological Review, 117(1), 300-6. doi:10.1037/a0018047

Bowers, J. S., Vankov, I. I., Damian, M. F., \& Davis, C. J. (2014). Neural networks learn highly selective representations in order to overcome the superposition catastrophe. Psychological review, 121(2), 248. doi:10.1037/a0035943

Buckley, M. J. (2005). The role of the perirhinal cortex and hippocampus in learning, memory, and perception. The Quarterly Journal of Experimental Psychology Section B, 58(34), 246-268.

Buckley, M. J., \& Gaffan, D. (2006). Perirhinal cortical contributions to object perception. Trends in Cognitive Sciences, 10(3), 100-7. doi:10.1016/j.tics.2006.01.008

Buckner, R. L., Andrews-Hanna, J. R., \& Schacter, D. L. (2008). The brain's default network: anatomy, function, and relevance to disease. Annals of the New York Academy of Sciences, 1124, 1-38. doi:10.1196/annals.1440.011

Bullier, J. (2001). Integrated model of visual processing. Brain Research Reviews, 36(2-3), 96-107. doi:10.1016/S0165-0173(01)00085-6 
Buneo, C. A., \& Andersen, R. A. (2006). The posterior parietal cortex: sensorimotor interface for the planning and online control of visually guided movements. Neuropsychologia, 44(13), 2594-2606. doi:10.1016/j.neuropsychologia.2005.10.011

Caporale, N., \& Dan, Y. (2008). Spike timing-dependent plasticity: a Hebbian learning rule. Annual Review of Neuroscience, 31, 25-46. doi:10.1146/annurev.neuro.31.060407.125639

Cavanagh, P., Hunt, A. R., Afraz, A., \& Rolfs, M. (2010). Visual stability based on remapping of attention pointers. Trends in Cognitive Sciences, 14(4), 147-53. doi:10.1016/j.tics.2010.01.007

Chklovskii, D. B., \& Koulakov, A. A. (2004). Maps in the brain: what can we learn from them? Annual Review of Neuroscience, 27, 369-92. doi:10.1146/annurev.neuro.27.070203.144226

Christoff, K., Keramatian, K., Gordon, A. M., Smith, R., \& Mädler, B. (2009). Prefrontal organization of cognitive control according to levels of abstraction. Brain Research, 1286, 94-105. doi:10.1016/j.brainres.2009.05.096

Cisek, P. (2007). Cortical mechanisms of action selection: the affordance competition hypothesis. Philosophical Transactions of the Royal Society of London. Series B, Biological Sciences, 362(1485), 1585-99. doi:10.1098/rstb.2007.2054

Cisek, P., \& Kalaska, J. F. (2010). Neural mechanisms for interacting with a world full of action choices. Annual Review of Neuroscience, 33(March), 269-98. doi:10.1146/annurev.neuro.051508.135409

Collins, J., \& Jin, D. Z. (2006). Grandmother cells and the storage capacity of the human brain. arXiv preprint q-bio/0603014.

Collins, J. A., \& Olson, I. R. (2014). Neuropsychologia Beyond the FFA : The role of the ventral anterior temporal lobes in face processing. Neuropsychologia, 1-15. doi:10.1016/j.neuropsychologia.2014.06.005

Cooper, R., \& Shallice, T. (2000). Contention scheduling and the control of routine activities. Cognitive neuropsychology, 17(4), 297-338.

Cooper, R. P., \& Shallice, T. (2006). Hierarchical schemas and goals in the control of sequential behavior. Psychological Review, 113(4), 887-916. doi:10.1037/0033295X.113.4.887

Corbetta, M., \& Shulman, G. L. (2002). Control of goal-directed and stimulusdriven attention in the brain. Nature Reviews. Neuroscience, 3(3), 201-15. doi:10.1038/nrn755

Coutanche, M. N., \& Thompson-Schill, S. L. (2014). Creating Concepts from Converging Features in Human Cortex. Cerebral Cortex. doi:10.1093/cercor/bhu057 
Cowell, R. a, Bussey, T. J., \& Saksida, L. M. (2010). Components of recognition memory: dissociable cognitive processes or just differences in representational complexity? Hippocampus, 20(11), 1245-62. doi:10.1002/hipo.20865

Crouzet, S. M., Kirchner, H., \& Thorpe, S. J. (2010). Fast saccades toward faces: face detection in just 100 ms. Journal of vision, 10(4), 16-16. doi:10.1167/10.4.16

Culham, J. C., Cavina-Pratesi, C., \& Singhal, A. (2006). The role of parietal cortex in visuomotor control: what have we learned from neuroimaging? Neuropsychologia, 44(13), 2668-84. doi:10.1016/j.neuropsychologia.2005.11.003

D'Souza, D., \& Karmiloff Smith, A. (2016). Why a developmental perspective is critical for understanding human cognition. Behavioral and Brain Sciences, 39.

Deco, G., \& Rolls, E. T. (2005). Attention, short-term memory, and action selection: a unifying theory. Progress in neurobiology, 76(4), 236-256.

Desimone, R., \& Duncan, J. (1995). Neural mechanisms of selective visual attention. Annual Review of Neuroscience, 18, 193-222. doi:10.1146/annurev.ne.18.030195.001205

DeWitt, I., \& Rauschecker, J. (2012). Phoneme and word recognition in the auditory ventral stream. Proceedings of the National Academy of Sciences, 109(8), 505514. doi:10.1073/pnas.1113427109

Diana, R. a, Yonelinas, A. P., \& Ranganath, C. (2007). Imaging recollection and familiarity in the medial temporal lobe: a three-component model. Trends in Cognitive Sciences, 11(9), 379-86. doi:10.1016/j.tics.2007.08.001

Dove, G. (2016). Three symbol ungrounding problems: Abstract concepts and the future of embodied cognition. Psychonomic bulletin \& review, 23(4), 1109-1121.

Driver, J., \& Noesselt, T. (2008). Multisensory interplay reveals crossmodal influences on "sensory-specific" brain regions, neural responses, and judgments. Neuron, 57(1), 11-23. doi:10.1016/j.neuron.2007.12.013

Duncan, J. (2006). EPS Mid-Career Award 2004: brain mechanisms of attention. Quarterly Journal of Experimental Psychology (2006), 59(1), 2-27. doi:10.1080/17470210500260674

Duncan, J., \& Humphreys, G. W. (1989). Visual Search and Stimulus Similarity. Psychological Review, 96(3), 433-458. doi:10.1037/0033-295X.96.3.433

Duncan, J., Humphreys, G., \& Ward, R. (1997). Competitive brain activity in visual attention. Current Opinion in Neurobiology, 7, 255-261. doi:10.1016/S09594388(97)80014-1

Duncan, J., Emslie, H., Williams, P., Johnson, R., \& Freer, C. (1996). Intelligence and the frontal lobe: The organization of goal-directed behavior. Cognitive psychology, 30(3), 257-303. doi:10.1006/cogp.1996.0008

Duncan, J., Parr, A., Woolgar, A., Thompson, R., Bright, P., Cox, S., ... NimmoSmith, I. (2008). Goal neglect and Spearman's g: competing parts of a complex task. 
Journal of Experimental Psychology. General, 137(1), 131-48. doi:10.1037/00963445.137.1.131

Engle, R. W. (2002). Working memory capacity as executive attention. Current directions in psychological science, 11(1), 19-23. doi: 10.1111/1467-8721.00160

Eliasmith, C. (1996). The third contender: a critical examination of the dynamicist theory of cognition. Philosophical Psychology, 9(4), 441-463.

Feldman, J., \& Ballard, D. (1982). Connectionist models and their properties. Cognitive Science, 254, 205-254. DOI: 10.1207/s15516709cog0603_1

Felleman, D., \& Essen, D. Van. (1991). Distributed hierarchical processing in the primate cerebral cortex. Cerebral Cortex, 1, 1-47.

Fernandez, D.N. (upcoming). Concrete vs absract: a failed dichotmy for cognition.

Franconeri, S. L., Alvarez, G. A., \& Cavanagh, P. (2013). Flexible cognitive resources: competitive content maps for attention and memory. Trends in Cognitive Sciences, 17(3), 134-141. doi:10.1016/j.tics.2013.01.010

Fuster, J. (1997). Network memory. Trends in Neurosciences, 20(10), 451-459. doi:10.1016/S0166-2236(97)01128-4

Fuster, J. M. (2000). Executive frontal functions. Experimental Brain Research, 133(1), 66-70. doi:10.1007/s002210000401

Fuster, J. M. (2003). Cortex and mind: Unifying cognition. New York: Oxford University Press.

Fuster, J. M. (2004). Upper processing stages of the perception-action cycle. Trends in cognitive sciences, 8(4), 143-145.

Fuster, J. M. (2006). The cognit: a network model of cortical representation. International Journal of Psychophysiology: Official Journal of the International Organization of Psychophysiology, 60(2), 125-32. doi:10.1016/j.ijpsycho.2005.12.015

Fuster, J. (2009). Cortex and memory: emergence of a new paradigm. Journal of Cognitive Neuroscience, 21(11), 2047-2072. doi:10.1162/jocn.2009.21280

Ghazanfar, A. A, \& Schroeder, C. E. (2006). Is neocortex essentially multisensory? Trends in Cognitive Sciences, 10(6), 278-85. doi:10.1016/j.tics.2006.04.008

Goodale, M. A. (2011). Transforming vision into action. Vision Research, 51(13), 1567-87. doi:10.1016/j.visres.2010.07.027

Goodale, M. A, \& Milner, A. (1992). Separate visual pathways for perception and action. Trends in Neurosciences, 15(1), 20-25. doi:10.1016/0166-2236(92)90344-8

Grafton, S. T., \& Hamilton, A. F. D. C. (2007). Evidence for a distributed hierarchy of action representation in the brain. Human Movement Science, 26(4), 590-616. doi:10.1016/j.humov.2007.05.009 
Graham, K. S., Lee, A. C., \& Barense, M. D. (2008). Invited Address at the Occasion of the Bertelson Award 2005 Impairments in visual discrimination in amnesia: Implications for theories of the role of medial temporal lobe regions in human memory. European Journal of Cognitive Psychology, 20(4), 655-696.

Graham, K. S., Barense, M. D., \& Lee, A. C. H. (2010). Going beyond LTM in the MTL: a synthesis of neuropsychological and neuroimaging findings on the role of the medial temporal lobe in memory and perception. Neuropsychologia, 48(4), 831-53. doi:10.1016/j.neuropsychologia.2010.01.001

Grainger, J. \& Jacobs, A.M. (1998). On localist connectionism and psychological science. In J. Grainger \& A.M. Jacobs (Eds.), Localist connectionist approaches to human cognition. Mawhah, NJ. : Erlbaum

Graziano, M. S. A. (2006). The organization of behavioral repertoire in motor cortex. Annual Review of Neuroscience, 29, 105-34. doi:10.1146/annurev.neuro.29.051605.112924

Graziano, M. (2010). Ethologically relevant movements mapped on the motor cortex. In A. a Ghazanfar \& M. L. Platt (Eds.), Primate neuroethology (pp. 454-470). New York, NY: Oxford University Press.

Graziano, M. S. (2016). Ethological action maps: a paradigm shift for the motor cortex. Trends in cognitive sciences, 20(2), 121-132. doi:10.1016/j.tics.2015.10.008

Graziano, M. S., \& Gross, C. G. (1998). Spatial maps for the control of movement. Current opinion in neurobiology, 8(2), 195-201. doi:10.1016/S0959-4388(98)80140-2

Gross, C. G. (2002). Genealogy of the "Grandmother Cell.” The Neuroscientist, 8(5), 512-518. doi:10.1177/107385802237175

Grossberg, S. (2013). Adaptive Resonance Theory: how a brain learns to consciously attend, learn, and recognize a changing world. Neural Networks, 37, 1-47. doi:10.1016/j.neunet.2012.09.017

Grossberg, S., \& Pearson, L. (2008). Laminar cortical dynamics of cognitive and motor working memory, sequence learning and performance: toward a unified theory of how the cerebral cortex works. Psychological Review, 115(3), 677-732. doi:10.1037/a0012618

Hamilton, A. F. D. C., \& Grafton, S. T. (2007). The motor hierarchy : from kinematics to goals and intentions Corresponding Author : In Y. Rossetti, M. Kawato, \& P. Haggard (Eds.), Attention \& Performance 22 The sensorimotor foundations of higher cognition (pp. 590-616). Oxford: Oxford University Press.

Hamker, F. H. (2005). The reentry hypothesis: the putative interaction of the frontal eye field, ventrolateral prefrontal cortex, and areas V4, IT for attention and eye movement. Cerebral Cortex (New York, N.Y. : 1991), 15(4), 431-47. doi:10.1093/cercor/bhh146

Harnad, S. (1990). The symbol grounding problem. Physica D: Nonlinear Phenomena, (42), 335-346. doi:10.1016/0167-2789(90)90087-6 
Hebb, D. O. (1949). The organization of behavior; a neuropsychological theory. New York, NY: Wiley.

Hubel, D. H., \& Wiesel, T. N. (1962). Receptive fields, binocular iteraction and functional archtecture in the cat's visual cortex. Journal of Physiology, 160, 106-154.

Hubel, D., \& Wiesel, T. (1968). Receptive fields and functional architecture of monkey striate cortex. The Journal of Physiology, 195(1), 215-243.

Nachev, P., \& Husain, M. (2006). Disorders of visual attention and the posterior parietal cortex. Cortex, 42(5), 766-773. doi:10.1016/S0010-9452(08)70415-5

Iacoboni, M. (2000). Attention and Sensorimotor integration Mapping the Embodied Mind. In A. W. Toga \& J. C. Mazziotta (Eds.), Brain mapping: The systems (pp. 463-490). San Diego: CA: Academic Press.

Kaas, J., Hackett, T., \& Tramo, M. (1999). Auditory processing in primate cerebral cortex. Current Opinion in Neurobiology, 9, 164-170. doi:10.1016/S0959-4388(99)800221

Karmiloff-Smith, A. (1998). Development itself is the key to understanding developmental disorders. Trends in Cognitive Sciences, 2(10), 389-98. doi:10.1016/S1364-6613(98)01230-3

Kirchner, H., \& Thorpe, S. J. (2006). Ultra-rapid object detection with saccadic eye movements: visual processing speed revisited. Vision Research, 46(11), 1762-76. doi:10.1016/j.visres.2005.10.002

Knierim, J. J., \& Van Essen, C. (1992). Neuronal Responses to Static Texture Patterns in Area Vl of the Alert Macaque Monkey. Journal of Neurophysiology, 67(4), 961-980.

Koechlin, E., \& Jubault, T. (2006). Broca's area and the hierarchical organization of human behavior. Neuron, 50(6), 963-74. doi:10.1016/j.neuron.2006.05.017

Koechlin, E., Ody, C., \& Kouneiher, F. (2003). The architecture of cognitive control in the human prefrontal cortex. Science, 302(November), 1181-1185. doi:10.1126/science.1088545

Koechlin, E., \& Summerfield, C. (2007). An information theoretical approach to prefrontal executive function. Trends in Cognitive Sciences, 11(6), 229-35. doi:10.1016/j.tics.2007.04.005

Koelewijn, T., Bronkhorst, A., \& Theeuwes, J. (2010). Attention and the multiple stages of multisensory integration: A review of audiovisual studies. Acta Psychologica, 134(3), 372-84. doi:10.1016/j.actpsy.2010.03.010

Kosslyn, S. M. (2006). You can play 20 questions with nature and win: Categorical versus coordinate spatial relations as a case study. Neuropsychologia, 44(9), 1519-1523. doi:10.1016/j.neuropsychologia.2006.01.022 
Kreiman, G., Koch, C., \& Fried, I. (2000). Category-specific visual responses of single neurons in the human medial temporal lobe. Nature Neuroscience, 3(9). doi:10.1038/78868

Kveraga, K., Boshyan, J., \& Bar, M. (2007). Magnocellular projections as the trigger of top-down facilitation in recognition. Journal of Neuroscience, 27(48), 13232-13240. doi:10.1523/JNEUROSCI.3481-07.2007

Kveraga, K., Ghuman, A. S., \& Bar, M. (2007). Top-down predictions in the cognitive brain. Brain and Cognition, 65(2), 145-68. doi:10.1016/j.bandc.2007.06.007

Lamme, V. A., Super, H., \& Spekreijse, H. (1998). Feedforward, horizontal, and feedback processing in the visual cortex. Current opinion in neurobiology, 8(4), 529-535. doi:10.1016/S0959-4388(98)80042-1

Lamme, V., \& Roelfsema, P. (2000). The distinct modes of vision offered by feedforward and recurrent processing. Trends in Neurosciences, 23(11), 571-579. doi:10.1016/S0166-2236(00)01657-X

Leaver, A., \& Rauschecker, J. (2010). Cortical representation of natural complex sounds: effects of acoustic features and auditory object category. The Journal of Neuroscience, 30(22), 7604-7612. doi:10.1523/JNEUROSCI.0296-10.2010

Lee, A. C. H., Yeung, L.-K., \& Barense, M. D. (2012). The hippocampus and visual perception. Frontiers in Human Neuroscience, 6(April), 91. doi:10.3389/fnhum.2012.00091

Lin, L., Chen, G., Kuang, H., Wang, D., \& Tsien, J. Z. (2007). Neural encoding of the concept of nest in the mouse brain. Proceedings of the National Academy of Sciences, 104(14), 6066-6071. doi: 10.1073/pnas.0701106104

Macdonald, C. J., Lepage, K. Q., Eden, U. T., \& Eichenbaum, H. (2011). Hippocampal "time cells" bridge the gap in memory for discontiguous events. Neuron, 71(4), 737-749. doi:10.1016/j.neuron.2011.07.012

Manns, J., Howard, M., \& Eichenbaum, H. (2007). Gradual changes in hippocampal activity support remembering the order of events. Neuron, 56, 530-540. doi:10.1016/j.neuron.2007.08.017

Mayor, J., \& Plunkett, K. (2012). How many neurons for your 'Grandmother'? Three arguments for localised representations. In N. Miyake, \& D. \&. C. Peebles R.P. (Eds.), Building bridges across cognitive sciences around the world. proceedings of the 34th annual conference of the cognitive science society (pp. 1990-1995). Austin, TX: Cognitive Science Society. Retrieved from http://archive-ouverte.unige.ch/unige:22687

McClelland, J. L., Botvinick, M. M., Noelle, D. C., Plaut, D. C., Rogers, T. T., Seidenberg, M. S., \& Smith, L. B. (2010). Letting structure emerge: connectionist and dynamical systems approaches to cognition. Trends in cognitive sciences, 14(8), 348-356. doi:10.1016/j.tics.2010.06.002 
McClelland, J. L., \& Rogers, T. T. (2003). The parallel distributed processing approach to semantic cognition. Nature Reviews. Neuroscience, 4(4), 310-22. doi:10.1038/nrn1076

McClelland, J., \& Rumelhart, D. (1981). An Interactive Activation Model of Context Effects in Letter Perception: Part 1. An Account of Basic Findings. Psychological Review, 88(5), 375-407.

Miller, E., \& Cohen, J. (2001). An integrative theory of prefrontal cortex function. Annual Review of Neuroscience, 24, 167-202. doi:10.1146/annurev.neuro.24.1.167

Miller, E. K., \& Wallis, J. D. (2009). Executive function and higher-order cognition: definition and neural substrates. Encyclopedia of neuroscience, 4(99-104).

Mongillo, G., Barak, O., \& Tsodyks, M. (2008). Synaptic Theory of Working Memory. Science, 319, 1543-1546. doi:10.1126/science.1150769

Mountcastle, V. (1997). The columnar organization of the neocortex. Brain, 120, 701-722. doi:10.1093/brain/120.4.701

Murray, E. A., Bussey, T. J., \& Saksida, L. M. (2007). Visual perception and memory: a new view of medial temporal lobe function in primates and rodents. Annual Review of Neuroscience, 30(February), 99-122. doi:10.1146/annurev.neuro.29.051605.113046

Murray, E. A., \& Bussey, T. J. (1999). Perceptual-mnemonic functions of the perirhinal cortex. Trends in Cognitive Sciences, 3(4), 142-151. doi:10.1016/S13646613(99)01303-0

Murthy, V. N. (2011). Olfactory maps in the brain. Annual Review of Neuroscience, 34, 233-58. doi:10.1146/annurev-neuro-061010-113738

Naya, Y., \& Suzuki, W. a. (2011). Integrating what and when across the primate medial temporal lobe. Science (New York, N.Y.), 333(6043), 773-6. doi:10.1126/science.1206773

Newcombe, N. S. (2011). What Is Neoconstructivism? Child Development Perspectives, 5(3), 157-160. doi:10.1111/j.1750-8606.2011.00180.x

Newell, A. (1973). You can't play 20 questions with nature and win: Projective comments on the papers of this symposium. In W. G. Chase (Ed.), Visual information processing. New York, NY: Academic Press.

Newell, A. (1990). Unified theories of cognition. Harvard University Press.

Okada, K., Rong, F., Venezia, J., Matchin, W., Hsich, I.-H., Saberi, K., ... Hickok, G. (2010). Hierarchical organization of human auditory cortex: evidence from acoustic invariance in the response to intelligible speech. Cerebral Cortex, 20, 2486-2495. doi:10.1093/cercor/bhp318 
Paz, R., Gelbard-Sagiv, H., Mukamel, R., Harel, M., Malach, R., \& Fried, I. (2010). A neural substrate in the human hippocampus for linking successive events. Proceedings of the National Academy of Sciences of the United States of America, 107(13), 6046-51. doi:10.1073/pnas.0910834107

Perrett, D., Rolls, E., \& Caan, W. (1982). Visual neurons responsive to faces in the monkey temporal cortex. Experimental Brain Research, 47(3), 329-342. doi:10.1007/BF00239352

Pierrot-deseilligny, C., Milea, D., \& Mu, M. (2004). Eye movement control by the cerebral cortex. Current Opinion in Neurology, 17, 17-25. doi:10.1097/01.wco.0000113942.12823.e0

Plaut, D. C., \& McClelland, J. L. (2010). Locating object knowledge in the brain: comment on Bowers's (2009) attempt to revive the grandmother cell hypothesis. Psychological Review, 117(1), 284-8. doi:10.1037/a0017101

Poggio, T., \& Bizzi, E. (2004). Generalization in vision and motor control. Nature, 431(October), 768-774. doi:doi.10.1038/nature03014

Pollen, D. A. (1999). Feature Article On the Neural Correlates of Visual Perception. Cerebral Cortex, 9, 4-19. doi: 10.1093/cercor/9.1.4

Posner, M. I., \& Petersen, S. E. (1990). The attention system of the human brain. Annual Review of Neuroscience, 13, 25-42.

Posner, M. I., \& Rothbart, M. K. (2007). Research on attention networks as a model for the integration of psychological science. Annual Review of Psychology, 58, $1-$ 23. doi:10.1146/annurev.psych.58.110405.085516

Quian Quiroga, R. (2012). Concept cells: the building blocks of declarative memory functions. Nature Reviews. Neuroscience, 13(8), 587-97. doi:10.1038/nrn3251

Quian Quiroga, R., Reddy, L., Koch, C., \& Fried, I. (2007). Decoding visual inputs from multiple neurons in the human temporal lobe. Journal of Neurophysiology, 98(19972007).

Quian Quiroga, R., Reddy, L., Kreiman, G., Koch, C., \& Fried, I. (2005). Invariant visual representation by single neurons in the human brain. Nature, 435(7045), 1102-7. doi:10.1038/nature03687

Ranganath, C. (2010). A unified framework for the functional organization of the medial temporal lobes and the phenomenology of episodic memory. Hippocampus, 20(11), 1263-90. doi:10.1002/hipo.20852

Rauschecker, J. P., \& Scott, S. K. (2009). Maps and streams in the auditory cortex: nonhuman primates illuminate human speech processing. Nature neuroscience, 12(6), 718-724. doi:10.1038/nn.2331 
Rey, H. G., Ison, M. J., Pedreira, C., Valentin, A., Alarcon, G., Selway, R., ... \& Quian Quiroga, R. (2015). Single-cell recordings in the human medial temporal lobe. Journal of anatomy, 227(4), 394-408. doi:10.1111/joa.12228

Riesenhuber, M., \& Poggio, T. (1999). Hierarchical models of object recognition in cortex. Nature Neuroscience, 2(11), 1019-1025. doi:10.1038/14819

Riesenhuber, M., \& Poggio, T. (2000). Models of object recognition. Nature Neuroscience, 3, 1199-1204. doi:10.1038/81479

Ritter, H., \& Kohonen, T. (1989). Biological Cybernetics Self-Organizing Semantic Maps. Biological Cybernetics, 61, 241-254. doi:10.1007/BF00203171

Rizzolatti, G., \& Craighero, L. (2004). The mirror-neuron system. Annual Review of Neuroscience, 27, 169-192. doi:10.1146/annurev.neuro.27.070203.144230

Rizzolatti, G., \& Craighero, L. (2010). Premotor theory of attention. Scholarpedia. doi:10.4249/scholarpedia.6311

Rizzolatti, G., \& Luppino, G. (2001). The cortical motor system. Neuron, 31, 889901. doi:10.1016/S0896-6273(01)00423-8

Rizzolatti, G., \& Matelli, M. (2003). Two different streams form the dorsal visual system: anatomy and functions. Experimental brain research, 153(2), 146-157. doi:10.1007/s00221-003-1588-0

Rizzolatti, G., Riggio, L., Dascola, I., \& Umiltá, C. (1987). Reorienting attention across the horizontal and vertical meridians: evidence in favor of a premotor theory of attention. Neuropsychologia, 25(1A), 31-40. doi:10.1016/0028-3932(87)90041-8

Rolls, E. (2008). Memory, attention, and decision-making: a unifying computational neuroscience approach. Oxford: Oxford University Press.

Rosch, E. (1978). Principles of Categorization. In E. Rosch and B. Lloyd (Eds.), Cognition and Categorization (pp. 27-48). Hillsdale, NJ: Lawrence Erlbaum Associates.

Roy, A. (2012). A theory of the brain: localist representation is used widely in the brain. Frontiers in Psychology, 3(December), 10-13. doi:10.3389/fpsyg.2012.00551

Roy, A. (2013). An extension of the localist representation theory: grandmother cells are also widely used in the brain. Frontiers in Psychology, 4(May), 300. doi:10.3389/fpsyg.2013.00300

Roy, A. (2015). On findings of category and other concept cells in the brain: Some theoretical perspectives on mental representation. Cognitive Computation, 7(3), 279-284. doi:10.1007/s12559-014-9307-7

Roy, A. (2017). The theory of localist representation and of a purely abstract cognitive system: the evidence from cortical columns, category cells, and multisensory neurons. Frontiers in psychology, 8, 186. doi: 10.3389/fpsyg.2017.00186 
Saito, N., Mushiake, H., Sakamoto, K., Itoyama, Y., \& Tanji, J. (2005). Representation of immediate and final behavioral goals in the monkey prefrontal cortex during an instructed delay period. Cerebral Cortex, 15(10), 1535-46. doi:10.1093/cercor/bhi032

Saksida, L. M., \& Bussey, T. J. (2010). The representational-hierarchical view of amnesia: translation from animal to human. Neuropsychologia, 48(8), 2370-84. doi:10.1016/j.neuropsychologia.2010.02.026

Sala, J. B., Rämä, P., \& Courtney, S. M. (2003). Functional topography of a distributed neural system for spatial and nonspatial information maintenance in working memory. Neuropsychologia, 41(3), 341-56. doi:10.1016/S0028-3932(02)00166-5

Schall, J. D. (2002). The neural selection and control of saccades by the frontal eye field. Philosophical Transactions of the Royal Society of London. Series B, Biological Sciences, 357(1424), 1073-82. doi:10.1098/rstb.2002.1098

Sedda, A., \& Scarpina, F. (2012). Dorsal and ventral streams across sensory modalities. Neuroscience bulletin, 28(3), 291-300. doi:10.1007/s12264-012-1223-9

Seidenberg, M. S., \& Plaut, D. C. (2006). Progress in understanding wordreading: Data fitting versus theory building. In S. Andrews (Ed.), From inkmarks to ideas: Current issues in lexical processing. Hove, England:Psychology Press.

Serences, J. T., \& Boynton, G. M. (2007). Feature-based attentional modulations in the absence of direct visual stimulation. Neuron, 55(2), 301-12. doi:10.1016/j.neuron.2007.06.015

Serre, T., Oliva, A., \& Poggio, T. (2007). A feedforward architecture accounts for rapid categorization. Proceedings of the National Academy of Sciences, 104(15), 64246429. doi: 10.1073/pnas.0700622104

Sève, L., \& Guespin-Michel, J. (2005). Émergence, complexité et dialectique : sur les systèmes dynamiques non linéaires [Emergence, complexity and dialectics: on nonlinear dynamic systems]. Paris: Odile Jacob.

Sève, Lucien (ed.) 1998. Sciences et dialectiques de la nature [Sciences and dialectics of nature]. Paris: La Dispute.

Shallice, T. (1982). Specific impairments of planning. Philosophical Transactions of the Royal Society of London B: Biological Sciences, 298(1089), 199-209. doi:10.1098/rstb.1982.0082

Shima, K., Isoda, M., Mushiake, H., \& Tanji, J. (2007). Categorization of behavioural sequences in the prefrontal cortex. Nature, 445(7125), 315-8. doi:10.1038/nature05470

Shipp, S. (2003). The functional logic of cortico-pulvinar connections. Philosophical Transactions of the Royal Society of London. Series B, Biological Sciences, 358(1438), 1605-24. doi:10.1098/rstb.2002.1213 
Shipp, S. (2004). The brain circuitry of attention. Trends in Cognitive Sciences, 8(5), 223-30. doi:10.1016/j.tics.2004.03.004

Silver, M., \& Kastner, S. (2009). Topographic maps in human frontal and parietal cortex. Trends in Cognitive Sciences, 13(11), 488-95. doi:10.1016/j.tics.2009.08.005

Simmons, W. K., \& Barsalou, L. W. (2003). The similarity-in-topography principle: reconciling theories of conceptual deficits. Cognitive Neuropsychology, 20(3), 451-86. doi:10.1080/02643290342000032

Simmons, W. K., \& Martin, A. (2009). The anterior temporal lobes and the functional architecture of semantic memory. Journal of the International Neuropsychological Society, 15(5), 645-649. doi:10.1017/S1355617709990348

Sirois, S., Spratling, M., Thomas, M. S. C., Westermann, G., Mareschal, D., \& Johnson, M. H. (2008). Précis of neuroconstructivism: how the brain constructs cognition. The Behavioral and Brain Sciences, 31(3), 321-31; discussion 331-56. doi:10.1017/S0140525X0800407X

Spence, C. (2013). Just how important is spatial coincidence to multisensory integration ? Evaluating the spatial rule. Annals of the New York Academy of Sciences, 1296, 31-49. doi:10.1111/nyas.12121

Spivey, M., \& Dale, R. (2006). Continuous dynamics in real-time cognition. Current Directions in Psychological Science, 15(5), 207-211. doi:10.1111/j.14678721.2006.00437.x

Squire, L. R. (2004). Memory systems of the brain: a brief history and current perspective. Neurobiology of Learning and Memory, 82(3), 171-7. doi:10.1016/j.nlm.2004.06.005

Squire, L. R., Stark, C. E. L., \& Clark, R. E. (2004). The medial temporal lobe. Annual Review of Neuroscience, 27, 2796. doi:10.1146/annurev.neuro.27.070203.144130

Stein, B. E., Jiang, W., \& Stanford, T. R. (2004). Multisensory integration in single neurons in the midbrain. In G. a Calvert, C. Spence, \& B. E. Stein (Eds.), The Handbook of Multisensory Processes (pp. 243-264). MIT Press.

Stein, B. E., \& Stanford, T. R. (2008). Multisensory integration: current issues from the perspective of the single neuron. Nature Reviews. Neuroscience, 9(4), 255-66. doi:10.1038/nrn2331

Stein, B. E., \& Stanford, T. R. (2013). Development of the Superior Colliculus/Optic Tectum. Neural Circuit Development and Function in the Healthy and Diseased Brain: Comprehensive Developmental Neuroscience, 3, 41.

Tanaka, K. (1996). Inferotemporal cortex and object vision. Annual Review of Neuroscience, 19, 109-39. doi:10.1146/annurev.ne.19.030196.000545 
Tanaka, K. (1997). Mechanisms of visual object recognition: monkey and human studies. Current Opinion in Neurobiology, 7(4), 523-9. doi:10.1016/S09594388(97)80032-3

Tanji, J., Shima, K., \& Mushiake, H. (2007). Concept-based behavioral planning and the lateral prefrontal cortex. Trends in Cognitive Sciences, 11(12), 528-34. doi:10.1016/j.tics.2007.09.007

Theeuwes, J., \& Kramer, A. (1998). Our eyes do not always go where we want them to go: Capture of the eyes by new objects. Psychological Science, 9(5), 379-385. doi:10.1111/1467-9280.00071

Thelen, E. (1995). Time-scale dynamics and the development of an embodied cognition. In R. F. Port \& T. Van Gelder (Eds.), Mind as motion: Explorations in the dynamics of cognition (pp. 69-100). Cambridge, MA, US: MIT Press.

Thomas, M. S., \& McClelland, J. L. (2008). Connectionist models of cognition. The Cambridge handbook of computational psychology, 23-58.

Thorpe, S. (2011). Grandmother Cells and Distributed Representations. In N. Kriegeskorte \& G. Kreiman (Eds.), Understanding visual population codes. Toward a common multivariate framework for cell recording and functional imaging. MIT Press.

Tian, B., Reser, D., Durham, A., Kustov, A., \& Rauschecker, J. (2001). Functional specialization in rhesus monkey auditory cortex. Science, 292, 290-293. doi:10.1126/science.1058911

Tipper, S. P., Lortie, C., \& Baylis, G. C. (1992). Selective reaching: evidence for action-centered attention. Journal of Experimental Psychology: Human Perception and Performance, 18(4), 891. doi:10.1037/0096-1523.18.4.891

Torralbo, A., \& Beck, D. (2008). Perceptual-load-induced selection as a result of local competitive interactions in visual cortex. Psychological Science, 19(10), 1045-1050. doi: 10.1111/j.1467-9280.2008.02197.x

Trappenberg, T. (2009). Fundamentals of computational neuroscience. OUP Oxford.

Trappenberg, T. P., Dorris, M. C., Munoz, D. P., \& Klein, R. M. (2001). A model of saccade initiation based on the competitive integration of exogenous and endogenous signals in the superior colliculus. Cognitive Neuroscience, Journal of, 13(2), 256-271. doi:10.1162/089892901564306

Tsao, D. Y., Freiwald, W. A., Tootell, R. B. H., \& Livingstone, M. S. (2006). A cortical region consisting entirely of face-selective cells. Science, 311(5761), 670-4. doi:10.1126/science.1119983

Uexküll, J. von. (1926). Theoretical Biology. Harcourt: Brace \& Co.

Uithol, S., Van Rooij, I., Bekkering, H., \& Haselager, P. (2012). Hierarchies in action and motor control. Journal of Cognitive Neuroscience, 24(5), 1077-1086. doi:10.1162/jocn_a_00204 
Uttal, W. R. (2001). The new phrenology: The limits of localizing cognitive processes in the brain. Life and mind: Philosophical issues in biology and psychology. Cambridge, MA, US: MIT Press.

VanRullen, R. (2003). Visual saliency and spike timing in the ventral visual pathway. Journal of Physiology, Paris, 97(2-3), 365-77. doi:10.1016/j.jphysparis.2003.09.010

Vezoli, J., Gariel, M., Markov, N. T., Anderson, J. C., Douglas, R., Martin, K. A. C., ... Kennedy, H. (2010). Hierarchical order in the cortex. In Cinquième conférence plénière française de Neurosciences Computationnelles, "Neurocomp'10. Aug 2010, Lyon, France. Retrieved March 18, 2014, from http://www.hal.inserm.fr/hal00553406/document

Wagner, A. D., Shannon, B. J., Kahn, I., \& Buckner, R. L. (2005). Parietal lobe contributions to episodic memory retrieval. Trends in Cognitive Sciences, 9(9), 445-53. doi:10.1016/j.tics.2005.07.001

Wallis, J. D., Anderson, K. C., \& Miller, E. K. (2001). Single neurons in prefrontal cortex encode abstract rules. Nature, 411(6840), 953-956. doi:10.1038/35082081

Wang, Z., Kruijne, W., \& Theeuwes, J. (2012). Lateral interactions in the superior colliculus produce saccade deviation in a neural field model. Vision research, 62, 66-74. doi:10.1016/j.visres.2012.03.024

Wandell, B. a, Dumoulin, S. O., \& Brewer, A. a. (2007). Visual field maps in human cortex. Neuron, 56(2), 366-83. doi:10.1016/j.neuron.2007.10.012

Wong, C., \& Gallate, J. (2012). The function of the anterior temporal lobe : A review of the empirical evidence. Brain Research, 1449, 94-116. doi:10.1016/j.brainres.2012.02.017

Zucker, R., \& Regehr, W. (2002). Short-term synaptic plasticity. Annual Review of Physiology, 64, 355-405. doi:10.1146/annurev.physiol.64.092501.114547 